\title{
Hypersymmetric extensions of Maxwell-Chern-Simons gravity in $2+1$ dimensions
}

\author{
Ricardo Caroca, ${ }^{1, *}$ Patrick Concha ${ }^{1, \dagger}$ Javier Matulich, ${ }^{2, ٪}$ Evelyn Rodríguez, ${ }^{3, \S}$ and David Tempo $\odot^{4, \|}$ \\ ${ }^{1}$ Departamento de Matemática y Física Aplicadas, Universidad Católica de la Santísima Concepción, \\ Alonso de Ribera 2850, CP 4090541 Concepción, Chile \\ ${ }^{2}$ Université Libre de Bruxelles and International Solvay Institutes, \\ ULB-Campus Plaine CP231, B-1050 Brussels, Belgium \\ ${ }^{3}$ Departamento de Física, Universidad del Bío-Bío, Avenida Collao 1202, Casilla 5-C, \\ CP 4081112 Concepción, Chile \\ ${ }^{4}$ Departamento de Ciencias Matemáticas y Físicas, \\ Facultad de Ingeniería Universidad Católica de Temuco, CP 4780000, Chile
}

(Received 7 June 2021; accepted 2 August 2021; published 7 September 2021)

\begin{abstract}
We present a consistent way of coupling three-dimensional Maxwell-Chern-Simons gravity theory with massless spin- $\frac{5}{2}$ gauge fields. We first introduce the simplest hyper-Maxwell-Chern-Simons gravity generically containing two massless spin-2 fields coupled with a massless Majorana fermion of spin $\frac{5}{2}$ whose novel underlying superalgebra is explicitly constructed. We then present three alternative hypersymmetric extensions of the Maxwell algebra which are shown to emerge from the Inönü-Wigner contraction procedure of precise combinations of the $\mathfrak{o} \mathfrak{g} \mathfrak{p}(1 \mid 4)$ and the $\mathfrak{s} \mathfrak{p}(4)$ algebras. This allows us to construct distinct types of hyper-Maxwell-Chern-Simons theories that extend to include generically interacting nonpropagating spin-4 fields accompanied by one or two spin- $\frac{5}{2}$ gauge fields.
\end{abstract}

DOI: 10.1103/PhysRevD.104.064011

\section{INTRODUCTION}

Hypergravity was an early alternative to supergravity theory [1,2] proposed by Aragone and Deser [3] that deems a spin-5/2 field as the superpartner of the graviton. Although this proposal initially attracted some attention [3-6], it was promptly discarded due to the incompatibility of the minimal coupling between gravity (spin 2) and the spin-5/2 field with higher-spin (HS) gauge invariance. This obstruction relies on the fact that the HS gauge variation of the Einstein-Hilbert action is proportional to the Ricci tensor so that it cannot be canceled by means of the minimally coupled spin-5/2 field, which is instead proportional to the full Riemann tensor. Nonetheless, owing to the particular relationship between the Riemann tensor and the Ricci tensor in three spacetime dimensions, Aragone and

\footnotetext{
*rcaroca@ucsc.cl

†patrick.concha@ucsc.cl

*javier.matulich@ulb.be

\$ekrodriguez@ubiobio.cl

"jtempo@uct.cl
}

Published by the American Physical Society under the terms of the Creative Commons Attribution 4.0 International license. Further distribution of this work must maintain attribution to the author(s) and the published article's title, journal citation, and DOI. Funded by SCOAP .
Deser managed to formulate the first consistent interacting and nonpropagating HS theory [7].

In $2+1$ dimensions, anti-de Sitter hypergravity theory was studied in [8-10]. The theory is constructed in terms of a Chern-Simons (CS) action for two copies of the $\mathfrak{o} \mathfrak{g} \mathfrak{p}(4 \mid 1)$ superalgebra ${ }^{1}$ and contains a spin- 2 field, a spin- 4 field, and a spin-5/2 field. The asymptotic structure analysis of this model was performed in [10], where it was shown that its asymptotic symmetry algebra is given by two copies of the hypersymmetric extension of the $W(2,4)$ algebra, known as the $W B_{2}$ superalgebra [20] and the $W(2,5 / 2,4)$ superalgebra [21]. Furthermore, this study also revealed the existence of hypersymmetry bounds involving a nonlinear function of the mass, angular momentum, and bosonic higher-spin charges, as well as an interesting class of HS solutions including solitons and extremal HS black holes with unbroken hypersymmetries $[10,22]$. In the vanishing cosmological constant limit it was verified that the spin-4 field decouples, thus reproducing the hypergravity theory studied in [23,24].

These results were explored more recently by means of an explicit extension of the Poincare algebra with

\footnotetext{
${ }^{1}$ Along these lines, (super)conformal gravity formulated as a CS gauge theory for $[\mathfrak{o} \mathfrak{g} p(1 \mid 4)] \mathfrak{s p}(4)$ (super)algebra was done in [11-15], while the corresponding asymptotic structure was studied in [16-19].
} 
half-integer spin generators in any dimension that allowed one to reformulate the hypergravity theory of Aragone and Deser as a genuine gauge theory using standard fiber bundle structure in terms of CS actions [25] in three and five spacetime dimensions $[23,24,26] .^{2}$ Indeed, the asymptotic structure analysis in $2+1$ dimensions led to a nonlinear hypersymmetric extension of the $\mathfrak{b m g}_{3}$ algebra, along with nonlinear bounds for the energy. Moreover, despite the fact that this theory involves HS fields, the absence of bosonic HS fields allows one to describe the theory in the standard Riemann-Cartan geometry. A natural question that then arises is whether it is possible to construct consistent extensions of the hypergravity theories based on extensions of the hyper-Poincaré algebra. It should be mentioned that, in order to improve the likelihood of this possibility, it is mandatory to have full control of the building blocks - namely, a consistent algebra with a nondegenerate invariant tensor. In what follows we address this question by considering a nontrivial extension of the Poincaré algebra that has long been known as the Maxwell algebra $[28,29]$, which is associated with the symmetry group of the Dirac (Klein-Gordon) equation minimally coupled to a constant electromagnetic field in Minkowski space in $3+1$ dimensions [30]. In any dimension, this algebra is characterized by the commutator

$$
\left[P_{a}, P_{b}\right]=Z_{a b}
$$

modifying in this way the commutator of the momentum generators, which vanishes for the Poincaré algebra. The study of different aspects of the Maxwell algebra in four or more dimensions, including its derivation as an $S$ expansion on the anti-de Sitter (AdS) algebra, can be found in [31,32]. The Maxwell group symmetries and its generalizations have been useful for extending standard general relativity through CS and Born-Infeld gravity theories in odd and even spacetime dimensions, respectively [33-36]. Deformations of this algebra and their dynamics through nonlinear realizations have been investigated [37-39], as have other interesting applications; see, e.g., [40-45].

In three spacetime dimensions, Maxwell-CS gravity appears to be a very appealing alternative theory of gravity in vacuum introduced in $[46-48]^{3}$ and subsequently studied in [54-56]. The asymptotic structure of the Maxwell-CS theory was investigated in [55] by imposing a set of suitable boundary conditions resulting in an asymptotic symmetry algebra given by a deformation of the $\mathfrak{b m}_{\mathfrak{3}}$ algebra, known to emerge from the asymptotic symmetry analysis of

\footnotetext{
${ }^{2}$ Fermionic HS generalizations of the Poincaré superalgebra were previously studied in [27] for any dimension. See [23] for further studies on these extensions of the hyper-Poincaré algebra in gauge theories beyond general relativity in odd dimensions [25].

${ }^{3}$ A CS gravity theory based on Maxwell algebra in $2+1$ was initially considered in $[46,47]$ as a prominent model leading to the two-dimensional linear gravities referred to in [49-53] as a dimensional reduction.
}

general relativity at null infinity [57-61], which goes in line with the result obtained in [62] by expanding the Virasoro algebra (see also [63,64]). Interestingly, the presence of the gravitational Maxwell gauge field modifies not only the asymptotic symmetry but also the vacuum of the theory [55]. Physical implications of the gravitational Maxwell gauge field have also been explored in the context of spin-3 gravity [65], nonrelativistic gravity [66-69], and supergravity [70,71]. It is worth noting that in three dimensions the extension of Poincare algebra found by Hietarinta [27] becomes isomorphic to three-dimensional Maxwell algebra that amounts to a simple interchanging of roles between the translation generators $P_{a}$ and $Z_{a}$ in the Maxwell algebra [72-74]. Indeed, CS gravity theories based on both the Hietarinta and Maxwell algebras were explored in [72,75], showing in particular that, upon spontaneous breaking of a local symmetry, they lead precisely to the topologically massive gravity theory [76] and the minimal massive gravity [77].

One of the main advantages of working in three spacetime dimensions is that the Maxwell algebra can alternatively be recovered as an Inönü-Wigner (IW) contraction of three copies of the $\mathfrak{S} \mathfrak{v}(2,1)$ algebra which also provides a nontrivial invariant form that is imperative for the construction of extensions of Maxwell-CS gauge theories [78]. In particular, the spin-3 Maxwell algebra as well as its corresponding invariant bilinear form can be derived by contracting three copies of the $\mathfrak{s l}(3, \mathbb{R})$ symmetry [65]. ${ }^{4}$ One may then ask whether a hypersymmetric extension of the Maxwell algebra with fermionic spin- $\frac{5}{2}$ generators, which transform in a spin- $\frac{3}{2}$ irreducible representation of the Lorentz group, can be obtained by contracting diverse combinations of the $\mathfrak{o} \mathfrak{s p}(1 \mid 4)$ and the $\mathfrak{s p}(4)$ algebras. In this work, we show that not one but three distinct hypersymmetric extensions of the Maxwell algebra, including their invariant bilinear forms, can be effectively derived through the IW procedure. The obtained hyper-Maxwell algebras indeed require the presence of spin- 4 generators and allow us to construct CS hypersymmetric gravity theories. Furthermore, we also show that a remarkable hyperMaxwell-CS gravity theory without spin-4 gauge fields can be constructed, and whose underlying superalgebra results in a sub-superalgebra of one of the hyper-Maxwell algebras that include spin-4 generators, which transform in an spin-3 irreducible representation of the Lorentz group.

The paper is organized as follows: In Sec. II, we briefly review the Maxwell-CS gravity theory defined on three spacetime dimensions. Sections III-V contain our main results. In Sec. III, we present the simplest CS hypergravity theory invariant under a hypersymmetric extension of the

\footnotetext{
${ }^{4}$ In [79-82] following suitable IW contractions allowed the authors to formulate the three-dimensional higher-spin gravity with vanishing cosmological constant as a CS gauge theory similar to their well-known counterparts in $\mathrm{AdS}_{3}$ [79,83-86].
} 
Maxwell algebra. In Sec. IV, we introduce three alternative hyper-Maxwell algebras including spin-4 generators by considering the IW contraction procedure. Section V is devoted to the construction of CS hypersymmetric gravity theories based on the aforementioned hyper-Maxwell symmetries. Section VI concludes our work with some discussions about future developments.

\section{THREE-DIMENSIONAL MAXWELL-CHERN- SIMONS GRAVITY THEORY}

In this section, we briefly review the three-dimensional Maxwell-CS gravity theory [46-48] (see also [54,55,70]). This alternative theory of gravity is based on the so-called Maxwell algebra, which can be seen as an extension and deformation of the Poincaré algebra $\mathfrak{i} \mathfrak{g}(2,1)$, which turns out to be a non-semisimple group. In addition to the usual local rotations $J_{a}$ and local translation generators $P_{a}$, the Maxwell symmetry is characterized by the presence of three additional Abelian generators $Z_{a}$. In particular, the Maxwell generators satisfy the following nonvanishing commutation relations:

$$
\begin{array}{ll}
{\left[J_{a}, J_{b}\right]=\epsilon_{a b}^{c} J_{c},} & {\left[J_{a}, P_{b}\right]=\epsilon_{a b}^{c} P_{c},} \\
{\left[J_{a}, Z_{b}\right]=\epsilon_{a b}^{c} Z_{c},} & {\left[P_{a}, P_{b}\right]=\epsilon_{a b}^{c} Z_{c},}
\end{array}
$$

where $a, b, c=0,1,2$ are Lorentz indices which are lowered and raised with the Minkowski metric $\eta_{a b}=(-1,1,1)$ and $\epsilon_{a b c}$ is the three-dimensional Levi-Civita tensor which satisfies $\epsilon_{012}=-\epsilon^{012}=1$.

The most general quadratic Casimir invariant for the Maxwell algebra is [46-48]

$$
C=\alpha_{0} J^{a} J_{a}+\alpha_{1} P^{a} J_{a}+\alpha_{2}\left(P^{a} P_{a}+J^{a} Z_{a}\right),
$$

where $\alpha_{0}, \alpha_{1}$, and $\alpha_{2}$ are arbitrary constants. The Maxwell algebra then admits the following nonvanishing components of the invariant tensor:

$$
\begin{array}{ll}
\left\langle J_{a} J_{b}\right\rangle=\alpha_{0} \eta_{a b}, \quad & \left\langle J_{a} P_{b}\right\rangle=\alpha_{1} \eta_{a b}, \\
\left\langle J_{a} Z_{b}\right\rangle=\alpha_{2} \eta_{a b}, & \left\langle P_{a} P_{b}\right\rangle=\alpha_{2} \eta_{a b} .
\end{array}
$$

The gauge connection one-form $A$ can be conveniently chosen as follows ${ }^{5}$ :

$$
A=\omega^{a} J_{a}+e^{a} P_{a}+k^{a} Z_{a},
$$

where $\omega^{a}$ is the (dualized) spin connection, $e^{a}$ denotes the dreibein and $k^{a}$ is the so-called gravitational Maxwell gauge field. The corresponding curvature two-form is given by

\footnotetext{
${ }^{5}$ More general choices of the gauge field can be considered leading to a more general Maxwell-CS theory that includes a nonvanishing torsion term.
}

$$
F=R^{a} J_{a}+T^{a} P_{a}+F^{a} Z_{a}
$$

where

$$
\begin{aligned}
R^{a} & =d \omega^{a}+\frac{1}{2} \epsilon_{b c}^{a} \omega^{b} \omega^{c}, \\
T^{a} & =d e^{a}+\epsilon^{a}{ }_{b c} \omega^{b} e^{c}, \\
F^{a} & =d k^{a}+\epsilon^{a}{ }_{b c} \omega^{b} k^{c}+\frac{1}{2} \epsilon_{b c}^{a} e^{b} e^{c} .
\end{aligned}
$$

Considering the gauge connection (2.4) and the invariant tensor (2.3), the corresponding action for the Maxwell algebra can then be described in terms of the threedimensional CS action,

$$
I_{C S}=\frac{k}{4 \pi} \int\left\langle A d A+\frac{2}{3} A^{3}\right\rangle,
$$

with $k=\frac{1}{4 G}$ being the CS level of the theory related to the gravitational constant $G$. Indeed, the action reads [46-48] (see also $[54,55,70]$ )

$$
I_{\text {Maxwell }}=\frac{k}{4 \pi} \int 2 \alpha_{1} R^{a} e_{a}+\alpha_{2}\left(e^{a} T_{a}+2 R^{a} k_{a}\right)+\alpha_{0} L(\omega),
$$

where

$$
L(\omega)=\left(d \omega^{a}+\frac{1}{3} \epsilon_{b c}^{a} \omega^{b} \omega^{c}\right) \omega_{a}
$$

is the Lorentz-Chern-Simons form.

The Maxwell-CS action contains three independent sectors proportional to $\alpha_{0}, \alpha_{1}$, and $\alpha_{2}$. The parity-odd term given by the Lorentz-CS three-form $[87,88]$ appears along the $\alpha_{0}$ constant while the Einstein-Hilbert term is related to the $\alpha_{1}$ constant. On the other hand, the additional gauge field $k^{a}$ contributes only to the $\alpha_{2}$ sector.

In particular, the equations of motion are given by

$$
\begin{aligned}
\delta \omega_{a}: \quad \alpha_{0} R^{a}+\alpha_{1} T^{a}+\alpha_{2} F^{a} & =0, \\
\delta k_{a}: \quad \alpha_{2} R^{a} & =0, \\
\delta e_{a}: \quad \alpha_{1} R^{a}+\alpha_{1} T^{a} & =0 .
\end{aligned}
$$

It follows that when $\alpha_{2} \neq 0$, the previous equations can be equivalently written as the vanishing of the curvature twoforms (2.6). It is worth mentioning that the suitable choice of the gauge field in Eq. (2.4) allows one to generically describe the theory in Riemannian geometry (torsionless). The standard $(2+1)$-dimensional gravity in vacuum is then recovered for the case $\alpha_{0}=\alpha_{2}=0$, which is the wellknown CS theory for $\mathfrak{i} \mathfrak{g} \mathfrak{o}(2,1)[87,88]$.

In this work, we will "hypersymmetrize" the threedimensional Maxwell gravity. As we will see, some 
hypersymmetric extensions will also require the presence of spin-4 gauge fields. The construction of the simplest hypersymmetric extension of the Maxwell-CS gravity is discussed in the next section.

\section{HYPER-MAXWELL-CHERN-SIMONS GRAVITY THEORY}

Here we present the simplest hypersymmetric extension of the Maxwell-CS gravity theory. To this end, we construct a hyper-Maxwell algebra by introducing fermionic generators which transform into an spin- $\frac{3}{2}$ irreducible representation of the Lorentz group. Therefore, the hyper-Maxwell algebra is spanned by the set $\left\{J_{a}, P_{a}, Z_{a}, Q_{\alpha a}\right\}$, whose generators satisfy the following nonvanishing (anti)commutation relations:

$$
\begin{aligned}
{\left[J_{a}, J_{b}\right] } & =\epsilon^{m}{ }_{a b} J_{m}, \quad\left[J_{a}, P_{b}\right]=\epsilon^{m}{ }_{a b} P_{m}, \\
{\left[J_{a}, Z_{b}\right] } & =\epsilon^{m}{ }_{a b} Z_{m}, \quad\left[P_{a}, P_{b}\right]=\epsilon^{m}{ }_{a b} Z_{m}, \\
{\left[J_{a}, Q_{\alpha b}\right] } & =\frac{1}{2}\left(\Gamma_{a}\right)_{\alpha}{ }_{\alpha} Q_{\beta b}+\epsilon_{a b c} Q_{\beta}^{c}, \\
\left\{Q_{\alpha a}, Q_{\beta b}\right\} & =-\frac{4}{3} \eta_{a b} Z_{c}\left(C \Gamma^{c}\right)_{\alpha \beta}+\frac{5}{3} \epsilon_{a b c} C_{\alpha \beta} Z^{c} \\
& +\frac{2}{3} Z_{(a \mid}\left(C \Gamma_{\mid b)}\right)_{\alpha \beta},
\end{aligned}
$$

where $Q_{\alpha a}$ are $\Gamma$-traceless vector-spinor generators that fulfill $\left(\Gamma_{a}\right)_{\alpha}^{\beta} Q_{a \beta}=\Gamma^{a} Q_{a}=0$. Here $C$ is the charge conjugation matrix

$$
C_{\alpha \beta}=\left(\begin{array}{cc}
0 & -1 \\
1 & 0
\end{array}\right)
$$

which satisfies $C^{T}=-C$ and $C \Gamma^{a}=\left(C \Gamma^{a}\right)^{T}$, with $\Gamma^{a}$ being the Dirac matrices in three spacetime dimensions. We shall denote this algebra as $\widehat{\mathfrak{h m}}$, which, like the hyperPoincaré algebra [23], requires neither an enlargement of the Lorentz group nor the introduction of bosonic HS generators to satisfy the Jacobi identities. One can notice that the algebra $\widehat{\mathfrak{h m}}$ has a structure similar to the so-called nonstandard Maxwell superalgebra [89,90], in which the translational generators $P_{a}$ are not expressed as bilinear expressions of fermionic generators. Nonetheless, since the sub-superalgebra spanned by the generators $J_{a}, Z_{a}$, and $Q_{\alpha a}$ is indeed the hyper-Poincaré algebra, as mentioned before, a simple interchanging of roles between the translation generators $P_{a}$ and $Z_{a}$ relates the superalgebra in Eq. (3.1) to its Hietarinta form in [27]. Therefore, one naturally expects gauge theories based on the hyperMaxwell or Hietarinta version of the algebra to certainly be endowed with quite different physical implications; see, e.g., [72-74]. In the present work, we will not consider this last reinterpretation of the theory since our purpose is to study the coupling of three-dimensional Maxwell gravity with massless spin- $\frac{5}{2}$ fields.

Let us now consider a hypersymmetric Maxwell-CS action that is invariant under the hyper-Maxwell algebra $\widehat{\mathfrak{h m}}$ (3.1). The CS action can be constructed from the gauge field

$$
A=e^{a} P_{a}+\omega^{a} J_{a}+k^{a} Z_{a}+\bar{\psi}^{a} Q_{a},
$$

whose components are the dreibein, the (dualized) spin connection, the gravitational Maxwell field and a Majorana spin- $\frac{5}{2}$ field. In particular, the Majorana conjugate reads $\bar{\psi}_{a \alpha}=\psi_{a}^{\beta} C_{\beta \alpha}$. The corresponding curvature two-form $F=$ $d A+\frac{1}{2}[A, A]$ is given by

$$
F_{\widehat{\mathfrak{h m}}}=T^{a} P_{a}+R^{a} J_{a}+\tilde{F}^{a} Z_{a}+D \bar{\psi}^{a} Q_{a}
$$

with

$$
\begin{aligned}
\tilde{F}^{a} & =F^{a}-\frac{3}{2} i \bar{\psi}_{b} \Gamma^{a} \psi^{b}, \\
D \psi^{a} & =d \psi^{a}+\frac{3}{2} \omega^{b} \Gamma_{b} \psi^{a}-\omega_{b} \Gamma^{a} \psi^{b},
\end{aligned}
$$

where $T^{a}, R^{a}$, and $F^{a}$ are as defined in Eq. (2.6). The fermionic fields are assumed to be $\Gamma$ traceless, i.e, $\Gamma^{a} \psi_{a}=0$. On the other hand, one can easily check to see that, in addition to $C_{0}=J^{a} J_{a}$ and $C_{1}=P^{a} J_{a}$, the hyper-Maxwell algebra $\widehat{\mathfrak{h m}}$ admits another quadratic Casimir invariant given by

$$
C_{2}=P^{a} P_{a}+J^{a} Z_{a}+Q_{\alpha}^{a} C^{\alpha \beta} Q_{\beta a}
$$

Hence, the $\widehat{\mathfrak{h m}}$ algebra admits the following nonvanishing components of an invariant tensor:

$$
\begin{array}{rlrl}
\left\langle J_{a} J_{b}\right\rangle & =\alpha_{0} \eta_{a b}, & \left\langle J_{a} P_{b}\right\rangle=\alpha_{1} \eta_{a b}, \\
\left\langle J_{a} Z_{b}\right\rangle & =\alpha_{2} \eta_{a b}, \quad\left\langle P_{a} P_{b}\right\rangle=\alpha_{2} \eta_{a b}, \\
\left\langle Q_{\alpha a} Q_{\beta b}\right\rangle & =2 \alpha_{2}\left(\frac{2}{3} C_{\alpha \beta} \eta_{a b}-\frac{1}{3} \epsilon_{a b c}\left(C \Gamma^{c}\right)_{\alpha \beta}\right),
\end{array}
$$

where $\alpha_{0}, \alpha_{1}$, and $\alpha_{2}$ are arbitrary constants. The invariance of such a bilinear form under the action of the hyperMaxwell algebra requires that $\left\langle J_{a} Z_{b}\right\rangle,\left\langle P_{a} P_{b}\right\rangle$, and $\left\langle Q_{\alpha a} Q_{\beta b}\right\rangle$ have the same global coefficient. When one uses the gauge connection one-form (3.3) and the invariant bilinear form (3.7) in the general form of a threedimensional CS action (2.7), it then reduces, up to boundary terms, to 


$$
\begin{aligned}
I_{\widehat{\mathfrak{h m}}}= & \frac{k}{4 \pi} \int 2 \alpha_{1} R^{a} e_{a}+\alpha_{2}\left(2 R^{a} k_{a}+T^{a} e_{a}+2 i \bar{\psi}_{a} D \psi^{a}\right) \\
& +\alpha_{0} L(\omega) .
\end{aligned}
$$

The previous CS action is invariant under the $\widehat{\mathfrak{h m}}$ algebra, extending the Maxwell gravity theory with a massless spin $-\frac{5}{2}$ gauge field. Note that the terms along the arbitrary constants $\alpha_{0}$ and $\alpha_{1}$ are not affected by the extension and coincide with the terms appearing in the bosonic action (2.8). The spin- $-\frac{5}{2}$ field only appears in the term proportional to the $\alpha_{2}$ constant. The action can then be seen as an "exotic" hypersymmetric gravity theory which does not require the presence of spin-4 gauge fields, which turns out to be as the natural Maxwellian extension of hypergravity given by Aragone and Deser in [7] but formulated as a genuine gauge theory. As we shall see later, there are other hypersymmetric extensions of the Maxwell gravity, but they require the presence of spin- 4 gauge fields.

For completeness, we provide the equations of motion, which are given as

$$
\begin{aligned}
\delta \omega_{a}: \quad \alpha_{0} R^{a}+\alpha_{1} T^{a}+\alpha_{2} \tilde{F}^{a} & =0 \\
\delta k_{a}: \quad \alpha_{2} R^{a} & =0 \\
\delta e_{a}: \quad \alpha_{1} R^{a}+\alpha_{1} T^{a} & =0 \\
\delta \bar{\psi}_{a}: \quad \alpha_{2} D \psi^{a} & =0 .
\end{aligned}
$$

When $\alpha_{2} \neq 0$, the previous equations can be equivalently written as the vanishing of the curvature two-forms (3.5). By construction, the CS action (3.8) is invariant under the local hypersymmetry transformation laws given by

$$
\begin{aligned}
\delta e^{a} & =0, \quad \delta \omega^{a}=0, \quad \delta k^{a}=3 i \bar{\epsilon}_{b} \Gamma^{a} \psi^{b}, \\
\delta \psi^{a} & =d \epsilon^{a}+\frac{3}{2} \omega^{b} \Gamma_{b} \epsilon^{a}-\omega_{b} \Gamma^{a} \epsilon^{b},
\end{aligned}
$$

where $\epsilon^{a}$ is the fermionic gauge parameter.

The CS action (3.8) is the simplest hypersymmetric extension of the Maxwell gravity without spin-4 gauge fields. As will be discussed, the previous hypersymmetric extension of the Maxwell algebra is not unique. However, to our knowledge, it seems that the hyper-Maxwell $\widehat{\mathfrak{h m}}$ is the only consistent way to accommodate spin- $-\frac{5}{2}$ generators to the Maxwell algebra without including spin-4 generators.

A different analysis can be done if we choose for the vierbein $e^{a}$ to accompany the $Z_{a}$ generator (or, equivalently, by performing $Z_{a} \leftrightarrow P_{a}$ ). The analysis will lead to a hypersymmetrization of the "Hietarinta gravity" [75] which, although interesting, is beyond the scope of this work.

The extension of the hyper-Maxwell gravity theory in Eq. (3.8) can then be extended to include fermionic fields of spin $(n+3 / 2)$ which become suitably described by a completely symmetric and (triple) $\Gamma$-traceless one-form $\bar{\psi}_{a_{1} \cdots a_{n}}$ so that the action reads

$$
\begin{aligned}
I_{\widehat{\mathfrak{h m}}}= & \frac{k}{4 \pi} \int 2 \alpha_{1} R^{a} e_{a}+\alpha_{2}\left(2 R^{a} k_{a}+T^{a} e_{a}+2 i \bar{\psi}_{a_{1} \cdots a_{n}} D \psi^{a_{1} \cdots a_{n}}\right) \\
& +\alpha_{0} L(\omega),
\end{aligned}
$$

with

$$
\begin{aligned}
D \psi^{a_{1} \cdots a_{n}}= & d \psi^{a_{1} \cdots a_{n}}+\left(n+\frac{1}{2}\right) \omega_{b} \Gamma^{b} \psi^{a_{1} \cdots a_{n}} \\
& -\omega_{b} \Gamma^{\left(a_{1}\right.} \psi^{\left.a_{2} \cdots a_{n}\right) b},
\end{aligned}
$$

and, being left invariant by the local hypersymmetry transformations, is given by

$$
\begin{gathered}
\delta e^{a}=0 \\
\delta \omega^{a}=0 \\
\delta k^{a}=2\left(n+\frac{1}{2}\right) i \bar{\epsilon}_{a_{1} \cdots a_{n}} \Gamma^{a} \psi^{a_{1} \cdots a_{n}}, \\
\delta \psi^{a_{1} \cdots a_{n}}=D \epsilon^{a_{1} \cdots a_{n}}
\end{gathered}
$$

Thus, the latter action naturally extends the hypergravity theory in [7] to include the Maxwell-CS gravity dynamics that amounts to describing two interacting nonpropagating gravitons and a spin- $(n+3 / 2)$ gauge field.

\section{ON THE EXTENSION OF THE MAXWELL ALGEBRA WITH SPIN $-\frac{5}{2}$ AND SPIN-4 GENERATORS}

In this section, we present three alternative hypersymmetric extensions of the Maxwell algebra, which are obtained through the IW contraction procedure [91]. As we shall see, such hyper-Maxwell algebras require the presence of spin- 4 generators and appear upon the contraction of diverse combinations of $\mathfrak{o} \mathfrak{g} \mathfrak{p}(1 \mid 4)$ and $\mathfrak{s p}(4)$ algebras. Interestingly, we show that the hyper-Maxwell algebra (3.1) without spin-4 generators (transforming in a spin-3 irreducible representation of the Lorentz group) appears as a subalgebra of one of the alternative hyperMaxwell algebras.

To start with, and in order to fix our notation, we will briefly review the $\mathfrak{o} \mathfrak{g} \mathfrak{p}(1 \mid 4)$ superalgebra.

\section{A. The $\mathfrak{o} \mathfrak{g} \mathfrak{p}(\mathbf{1} \mid \mathbf{4})$ superalgebra}

The $\mathfrak{o} \mathfrak{g} \mathfrak{p}(1 \mid 4)$ superalgebra with $\mathfrak{g l}(2 \mid R)$ principal embedded in $\mathfrak{s p}(4)$ is spanned by the set of generators $\left\{T_{a}, T_{a b c}, \mathcal{G}_{\alpha a}\right\}$, which satisfy the following nonvanishing (anti)commutation relations: 


$$
\begin{aligned}
{\left[T_{a}, T_{b}\right]=} & \epsilon^{m}{ }_{a b} T_{m}, \\
{\left[T_{a}, T_{b c d}\right]=} & 3 \epsilon^{m}{ }_{a(b} T_{c d) m}, \\
{\left[T_{a}, \mathcal{G}_{\alpha b}\right]=} & \frac{1}{2}\left(\Gamma_{a}\right)_{\alpha}^{\beta} \mathcal{G}_{\beta b}+\epsilon_{a b c} \mathcal{G}_{\beta}^{c}, \\
{\left[T_{a b c}, T_{m n k}\right]=} & -6\left(\eta_{(a b} \epsilon_{c)(m}^{l} \eta_{n k)}+5 \epsilon_{(m \mid(a}^{l} \delta_{b}^{d} \eta_{c) \mid n} \eta_{k) d}\right) T_{l} \\
& +2\left(5 \epsilon_{(m \mid(a}^{l} \delta_{b}^{d} T_{c) l n} \eta_{k) d}-\epsilon_{(m \mid(a}^{l} \eta_{b c)} T_{\mid n k) l}\right. \\
& \left.-\epsilon_{(m(a \mid}^{l} T_{b c) l} \eta_{\mid n k)}\right), \\
{\left[T_{a b c}, \mathcal{G}_{\alpha d}\right]=} & \left(\delta^{k}{ }_{d} \eta_{(a b \mid}-5 \eta_{d(a} \delta_{b \mid}^{k}\right)\left(\Gamma_{\mid c)}\right)_{\alpha}^{\beta} \mathcal{G}_{\beta k} \\
& +\eta_{(a b \mid}\left(\Gamma_{d}\right)_{\alpha}{ }_{\alpha} \mathcal{G}_{\beta \mid c)}, \\
\left\{\mathcal{G}_{\alpha a}, \mathcal{G}_{\beta b}\right\}= & \left(T_{a b c}-\frac{4}{3} \eta_{a b} T_{c}\right)\left(C \Gamma^{c}\right)_{\alpha \beta}+\frac{5}{3} \epsilon_{a b c} C_{\alpha \beta} T^{c} \\
& +\frac{2}{3} T_{(a \mid}\left(C \Gamma_{\mid b)}\right)_{\alpha \beta},
\end{aligned}
$$

where $a, b, \ldots=0,1,2$ are Lorentz indices lowered and raised with the off-diagonal Minkowski metric $\eta_{a b}$ and $\epsilon_{b c}^{m}$ is the three-dimensional Levi-Civita tensor. Here $T_{a}$ span the $\mathfrak{g l}(2, R)$ subalgebra and stand for the spin-2 generators, while $T_{a b c}$ and the fermionic $\mathcal{G}_{\alpha a}$ generators yield, respectively, to spin- 4 and spin $-\frac{5}{2}$ fields in the Chern-Simons theory. Let us note that $T_{a b c}$ are traceless and totally symmetric generators satisfying $\eta^{a b} T_{a b c}=0$, while
$\mathcal{G}_{\alpha a}$ are $\Gamma$-traceless vector-spinor generators satisfying $\left(\Gamma^{a}\right)^{\beta}{ }_{\alpha} \mathcal{G}_{\beta a}=\Gamma^{a} \mathcal{G}_{a}=0$. In particular, the subalgebra spanned by the set $\left\{T_{a}, T_{a b c}\right\}$ defines a $\mathfrak{g} \mathfrak{p}(4)$ algebra.

The $\mathfrak{o} \mathfrak{s p}(1 \mid 4)$ superalgebra has the following nonvanishing components of the invariant tensor

$$
\begin{aligned}
\left\langle T_{a} T_{b}\right\rangle & =\frac{1}{2} \eta_{a b}, \\
\left\langle T_{a b c} T_{m n k}\right\rangle & =5 \eta_{m(a} \eta_{b \mid n} \eta_{\mid c) k}-3 \eta_{(a b} \eta_{\mid c)(m} \eta_{n k)}, \\
\left\langle\mathcal{G}_{\alpha a} \mathcal{G}_{\beta b}\right\rangle & =\frac{2}{3} C_{\alpha \beta} \eta_{a b}-\frac{1}{3} \epsilon_{a b c}\left(C \Gamma^{c}\right)_{\alpha \beta} .
\end{aligned}
$$

Let us note that the hyper-Poincaré algebra with and without spin-4 generators can be obtained by considering different IW contractions of the $\mathfrak{o} \mathfrak{g} \mathfrak{p}(1 \mid 4) \otimes \mathfrak{s p}(4)$ superalgebra [24]. Here we shall see that three alternative hyper-Maxwell algebras appear when we consider the IW contractions of the $\mathfrak{o} \mathfrak{s} \mathfrak{p}(1 \mid 4) \otimes \mathfrak{o} \mathfrak{s} \mathfrak{p}(1 \mid 4) \otimes \mathfrak{s} \mathfrak{p}(4)$ and $\mathfrak{o} \mathfrak{g} \mathfrak{p}(1 \mid 4) \otimes \mathfrak{s} \mathfrak{p}(4) \otimes \mathfrak{s p}(4)$ superalgebras.

\section{B. Hyper-Maxwell algebra with spin-4 generators}

Let us first consider the $\mathfrak{o} \mathfrak{s} \mathfrak{p}(1 \mid 4) \otimes \mathfrak{o} \mathfrak{g} \mathfrak{p}(1 \mid 4) \otimes \mathfrak{g} \mathfrak{p}(4)$ superalgebra. One can then show that the IW contraction of such a structure allows us to obtain a hyper-Maxwell algebra with spin-4 generators. To this end, let us consider the following redefinition of the generators:

$$
\begin{aligned}
& J_{a}=T_{a}+T_{a}^{+}+T_{a}^{-}, \quad P_{a}=\frac{1}{\ell}\left(T_{a}^{+}-T_{a}^{-}\right), \quad Z_{a}=\frac{1}{\ell^{2}}\left(T_{a}^{+}+T_{a}^{-}\right), \\
& J_{a b c}=T_{a b c}+T_{a b c}^{+}+T_{a b c}^{-}, \quad P_{a b c}=\frac{1}{\ell}\left(T_{a b c}^{+}-T_{a b c}^{-}\right), \quad Z_{a b c}=\frac{1}{\ell^{2}}\left(T_{a b c}^{+}+T_{a b c}^{-}\right), \\
& Q_{\alpha a}=\frac{1}{\sqrt{\ell}}\left(\mathcal{G}_{\alpha a}^{+}-i \mathcal{G}_{\alpha a}^{-}\right), \quad \Sigma_{\alpha a}=\frac{1}{\sqrt{\ell^{3}}}\left(\mathcal{G}_{\alpha a}^{+}+i \mathcal{G}_{\alpha a}^{-}\right),
\end{aligned}
$$

where $\left\{T_{a}^{+}, T_{a b c}^{+}, \mathcal{G}_{\alpha a}^{+}\right\}$and $\left\{T_{a}^{-}, T_{a b c}^{-}, \mathcal{G}_{\alpha a}^{-}\right\}$each span an $\mathfrak{o} \mathfrak{s} \mathfrak{p}(1 \mid 4)$ superalgebra, while $\left\{T_{a}, T_{a b c}\right\}$ are $\mathfrak{s} \mathfrak{p}(4)$ generators. On the other hand, $\ell$ is a length parameter related to the cosmological constant through $\Lambda \propto \pm \frac{1}{\ell^{2}}$. It is simple to verify that the set of generators $\left\{J_{a}, P_{a}, Z_{a}, J_{a b c}, P_{a b c}, Z_{a b c}, Q_{\alpha a}, \Sigma_{\alpha a}\right\}$ satisfies a hypersymmetric version of the so-called AdS-Lorentz algebra [92-96]. It is then straightforward to show that the algebra obtained after the vanishing cosmological constant limit $\ell \rightarrow \infty$ leads to the following hypersymmetric algebra:

$$
\begin{array}{rlrl}
{\left[J_{a}, J_{b}\right]} & =\epsilon^{m}{ }_{a b} J_{m}, & {\left[J_{a}, P_{b}\right]} & =\epsilon^{m}{ }_{a b} P_{m}, \\
{\left[J_{a}, Z_{b}\right]} & =\epsilon^{m}{ }_{a b} Z_{m}, & {\left[P_{a}, P_{b}\right]} & =\epsilon^{m}{ }_{a b} Z_{m}, \\
{\left[J_{a}, J_{b c d}\right]} & =3 \epsilon^{m}{ }_{a(b} J_{c d) m}, & {\left[J_{a}, P_{b c d}\right]} & =3 \epsilon^{m}{ }_{a(b} P_{c d) m}, \\
{\left[P_{a}, J_{b c d}\right]} & =3 \epsilon^{m}{ }_{a(b} P_{c d) m}, & {\left[J_{a}, Z_{b c d}\right]} & =3 \epsilon^{m}{ }_{a(b} Z_{c d) m}, \\
{\left[Z_{a}, J_{b c d}\right]} & =3 \epsilon^{m}{ }_{a(b} Z_{c d) m}, & {\left[P_{a}, P_{b c d}\right]} & =3 \epsilon^{m}{ }_{a(b} Z_{c d) m}, \\
{\left[J_{a}, Q_{\alpha b}\right]} & =\frac{1}{2}\left(\Gamma_{a}\right)_{\alpha}^{\beta} Q_{\beta b}+\epsilon_{a b c} Q_{\beta}^{c}, \\
{\left[J_{a}, \Sigma_{\alpha b}\right]} & =\frac{1}{2}\left(\Gamma_{a}\right)_{\alpha}^{\beta} \Sigma_{\beta b}+\epsilon_{a b c} \Sigma_{\beta}^{c}, \\
{\left[P_{a}, Q_{\alpha b}\right]} & =\frac{1}{2}\left(\Gamma_{a}\right)_{\alpha}^{\beta} \Sigma_{\beta b}+\epsilon_{a b c} \Sigma_{\beta}^{c},
\end{array}
$$




$$
\begin{aligned}
& {\left[J_{a b c}, J_{m n k}\right]=-6\left(\eta_{(a b} \epsilon_{c)(m}^{l} \eta_{n k)}+5 \epsilon_{(m \mid(a}^{l} \delta_{b}^{d} \eta_{c) \mid n} \eta_{k) d}\right) J_{l}} \\
& +2\left(5 \epsilon_{(m \mid(a}^{l} \delta_{b}{ }_{b} J_{c) l \mid n} \eta_{k) d}-\epsilon_{(m \mid(a}^{l} \eta_{b c)} J_{\mid n k) l}-\epsilon_{(m(a \mid}^{l} J_{b c) l} \eta_{\mid n k)}\right), \\
& {\left[J_{a b c}, P_{m n k}\right]=-6\left(\eta_{(a b} \epsilon_{c)(m}^{l} \eta_{n k)}+5 \epsilon_{(m \mid(a}^{l} \delta_{b}^{d} \eta_{c) \mid n} \eta_{k) d}\right) P_{l}} \\
& +2\left(5 \epsilon_{(m \mid(a}^{l} \delta_{b}^{d} P_{c) l \mid n} \eta_{k) d}-\epsilon_{(m \mid(a}^{l} \eta_{b c)} P_{\mid n k) l}-\epsilon_{(m(a \mid}^{l} P_{b c) l} \eta_{\mid n k)}\right), \\
& {\left[J_{a b c}, Z_{m n k}\right]=-6\left(\eta_{(a b} \epsilon_{c)(m}^{l} \eta_{n k)}+5 \epsilon_{(m \mid(a}^{l} \delta_{b}^{d} \eta_{c) \mid n} \eta_{k) d}\right) Z_{l}} \\
& +2\left(5 \epsilon_{(m \mid(a}^{l} \delta_{b}^{d} Z_{c) l \mid n} \eta_{k) d}-\epsilon_{(m \mid(a}^{l} \eta_{b c)} Z_{\mid n k) l}-\epsilon_{(m(a \mid}^{l} Z_{b c) l} \eta_{\mid n k)}\right), \\
& {\left[P_{a b c}, P_{m n k}\right]=-6\left(\eta_{(a b} \epsilon_{c)(m}^{l} \eta_{n k)}+5 \epsilon_{(m \mid(a}^{l} \delta_{b}^{d} \eta_{c) \mid n} \eta_{k) d}\right) Z_{l}} \\
& +2\left(5 \epsilon_{(m \mid(a}^{l} \delta_{b}^{d} Z_{c) l \mid n} \eta_{k) d}-\epsilon_{(m \mid(a}^{l} \eta_{b c)} Z_{\mid n k) l}-\epsilon_{(m(a \mid}^{l} Z_{b c) l} \eta_{\mid n k)}\right), \\
& \left.\left[J_{a b c}, Q_{\alpha d}\right]=\left(\delta^{k}{ }_{d} \eta_{(a b \mid}-5 \eta_{d(a} \delta_{b \mid}^{k}\right)\left(\Gamma_{\mid c)}\right)_{\alpha}^{\beta} Q_{\beta k}+\eta_{(a b \mid}\left(\Gamma_{d}\right)^{\beta}{ }_{\alpha} Q_{\beta \mid c)}\right), \\
& {\left[J_{a b c}, \Sigma_{\alpha d}\right]=\left(\delta_{d}^{k} \eta_{(a b \mid}-5 \eta_{d(a} \delta_{b \mid}^{k}\right)\left(\Gamma_{\mid c)}\right)_{\alpha}^{\beta} \Sigma_{\beta k}+\eta_{(a b \mid}\left(\Gamma_{d}\right)^{\beta}{ }_{\alpha} \Sigma_{\beta \mid c)},} \\
& {\left[P_{a b c}, Q_{\alpha d}\right]=\left(\delta^{k}{ }_{d} \eta_{(a b \mid}-5 \eta_{d(a} \delta_{b \mid}^{k}\right)\left(\Gamma_{\mid c)}\right)_{\alpha}^{\beta} \Sigma_{\beta k}+\eta_{(a b \mid}\left(\Gamma_{d}\right)_{\alpha}^{\beta} \Sigma_{\beta \mid c)},} \\
& \left\{Q_{\alpha a}, Q_{\beta b}\right\}=\left(P_{a b c}-\frac{4}{3} \eta_{a b} P_{c}\right)\left(C \Gamma^{c}\right)_{\alpha \beta}+\frac{5}{3} \epsilon_{a b c} C_{\alpha \beta} P^{c}+\frac{2}{3} P_{(a \mid}\left(C \Gamma_{\mid b)}\right)_{\alpha \beta}, \\
& \left\{Q_{\alpha a}, \Sigma_{\beta b}\right\}=\left(Z_{a b c}-\frac{4}{3} \eta_{a b} Z_{c}\right)\left(C \Gamma^{c}\right)_{\alpha \beta}+\frac{5}{3} \epsilon_{a b c} C_{\alpha \beta} Z^{c}+\frac{2}{3} Z_{(a \mid}\left(C \Gamma_{\mid b)}\right)_{\alpha \beta} .
\end{aligned}
$$

The newly obtained algebra, which we shall denote as $\mathfrak{h m}_{(4)}$, corresponds to a hyper-Maxwell algebra in the presence of the spin-4 generators $\left\{J_{a b c}, P_{a b c}, Z_{a b c}\right\}$. This hypersymmetric extension of the Maxwell algebra is characterized by two fermionic generators $Q_{\alpha a}$ and $\Sigma_{\alpha a}$. The presence of a second spinorial charge is not arbitrary but is required to satisfy the Jacobi identities. Let us note that the presence of a second fermionic charge has already been discussed in the context of $D=11$ supergravity [97] and superstring theory [98]. Subsequently, it was also studied in the context of the supersymmetric extension of the
Maxwell algebra in [99-106]. As we will see in the next section, this novel algebra will allow us to construct a different Maxwell hypergravity theory in three spacetime dimensions.

\section{Nonstandard hyper-Maxwell algebra with spin-4 generators}

A different hyper-Maxwell algebra with one vectorspinor generator can be recovered from the $\mathfrak{o} \mathfrak{s p}(1 \mid 4) \otimes$ $\mathfrak{s p}(4) \otimes \mathfrak{s} \mathfrak{p}(4)$ superalgebra. Let us first consider the following redefinition of the generators:

$$
\begin{aligned}
& J_{a}=T_{a}+T_{a}^{+}+T_{a}^{-}, \quad P_{a}=\frac{1}{\ell}\left(T_{a}^{+}-T_{a}^{-}\right), \quad Z_{a}=\frac{1}{\ell^{2}}\left(T_{a}^{+}+T_{a}^{-}\right), \\
& J_{a b c}=T_{a b c}+T_{a b c}^{+}+T_{a b c}^{-}, \quad P_{a b c}=\frac{1}{\ell}\left(T_{a b c}^{+}-T_{a b c}^{-}\right), \quad Z_{a b c}=\frac{1}{\ell^{2}}\left(T_{a b c}^{+}+T_{a b c}^{-}\right), \\
& Q_{\alpha a}=\frac{\sqrt{2}}{\ell} \mathcal{G}_{\alpha a},
\end{aligned}
$$

where the subset $\left\{T_{a}^{+}, T_{a b c}^{+}, \mathcal{G}_{\alpha a}\right\}$ satisfies an $\mathfrak{o} \mathfrak{s} \mathfrak{p}(1 \mid 4)$ superalgebra, while $\left\{T_{a}^{-}, T_{a b c}^{-}\right\}$and $\left\{T_{a}, T_{a b c}\right\}$ each span an $\mathfrak{s p}(4)$ algebra. After considering the above redefinition, the set of generators $\left\{J_{a}, P_{a}, Z_{a}, J_{a b c}, P_{a b c}, Z_{a b c}, Q_{\alpha a}\right\}$ satisfies an alternative hypersymmetric version of the AdS-Lorentz algebra [92-96] whose flat limit leads to the following algebra: 


$$
\begin{aligned}
& {\left[J_{a}, J_{b}\right]=\epsilon_{a b}^{m} J_{m}, \quad\left[J_{a}, P_{b}\right]=\epsilon^{m}{ }_{a b} P_{m},} \\
& {\left[J_{a}, Z_{b}\right]=\epsilon^{m}{ }_{a b} Z_{m}, \quad\left[P_{a}, P_{b}\right]=\epsilon^{m}{ }_{a b} Z_{m},} \\
& {\left[J_{a}, J_{b c d}\right]=3 \epsilon^{m}{ }_{a(b} J_{c d) m}, \quad\left[J_{a}, P_{b c d}\right]=3 \epsilon^{m}{ }_{a(b} P_{c d) m},} \\
& {\left[P_{a}, J_{b c d}\right]=3 \epsilon_{a(b}^{m} P_{c d) m}, \quad\left[J_{a}, Z_{b c d}\right]=3 \epsilon_{a(b}^{m} Z_{c d) m},} \\
& {\left[Z_{a}, J_{b c d}\right]=3 \epsilon_{a(b}^{m} Z_{c d) m}, \quad\left[P_{a}, P_{b c d}\right]=3 \epsilon_{a(b}^{m} Z_{c d) m},} \\
& {\left[J_{a}, Q_{\alpha b}\right]=\frac{1}{2}\left(\Gamma_{a}\right)_{\alpha}^{\beta} Q_{\beta b}+\epsilon_{a b c} Q_{\beta}^{c},} \\
& {\left[J_{a b c}, J_{m n k}\right]=-6\left(\eta_{(a b} \epsilon_{c)(m}^{l} \eta_{n k)}+5 \epsilon_{(m \mid(a}^{l} \delta_{b}^{d} \eta_{c) \mid n} \eta_{k) d}\right) J_{l}} \\
& +2\left(5 \epsilon_{(m \mid(a}^{l} \delta_{b}^{d} J_{c) l \mid n} \eta_{k) d}-\epsilon_{(m \mid(a}^{l} \eta_{b c)} J_{\mid n k) l}-\epsilon_{(m \mid(a}^{l} J_{b c) l} \eta_{\mid n k)}\right), \\
& {\left[J_{a b c}, P_{m n k}\right]=-6\left(\eta_{(a b} \epsilon_{c)(m}^{l} \eta_{n k)}+5 \epsilon_{(m \mid(a}^{l} \delta_{b}^{d} \eta_{c) \mid n} \eta_{k) d}\right) P_{l}} \\
& +2\left(5 \epsilon_{(m \mid(a}^{l} \delta_{b}^{d} P_{c) l \mid n} \eta_{k) d}-\epsilon_{(m \mid(a}^{l} \eta_{b c)} P_{\mid n k) l}-\epsilon_{(m \mid(a}^{l} P_{b c) l} \eta_{\mid n k)}\right), \\
& {\left[J_{a b c}, Z_{m n k}\right]=-6\left(\eta_{(a b} \epsilon_{c)(m}^{l} \eta_{n k)}+5 \epsilon_{(m \mid(a}^{l} \delta_{b}^{d} \eta_{c) \mid n} \eta_{k) d}\right) Z_{l}} \\
& +2\left(5 \epsilon_{(m \mid(a}^{l} \delta_{b}^{d} Z_{c) l \mid n} \eta_{k) d}-\epsilon_{(m \mid(a}^{l} \eta_{b c)} Z_{\mid n k) l}-\epsilon_{(m \mid(a}^{l} Z_{b c) l} \eta_{\mid n k)}\right), \\
& {\left[P_{a b c}, P_{m n k}\right]=-6\left(\eta_{(a b} \epsilon_{c)(m}^{l} \eta_{n k)}+5 \epsilon_{(m \mid(a}^{l} \delta^{d}{ }_{b} \eta_{c) \mid n} \eta_{k) d}\right) Z_{l}} \\
& +2\left(5 \epsilon_{(m \mid(a}^{l} \delta_{b}^{d} Z_{c) l \mid n} \eta_{k) d}-\epsilon_{(m \mid(a}^{l} \eta_{b c)} Z_{\mid n k) l}-\epsilon_{(m \mid(a}^{l} Z_{b c) l} \eta_{\mid n k)}\right), \\
& {\left[J_{a b c}, Q_{\alpha d}\right]=\left(\delta^{k}{ }_{d} \eta_{(a b \mid}-5 \eta_{d(a} \delta_{b \mid}^{k}\right)\left(\Gamma_{\mid c)}\right)_{\alpha}^{\beta} Q_{\beta k}+\eta_{(a b \mid}\left(\Gamma_{d}\right)^{\beta}{ }_{\alpha} Q_{\beta \mid c)},} \\
& \left\{Q_{\alpha a}, Q_{\beta b}\right\}=\left(Z_{a b c}-\frac{4}{3} \eta_{a b} Z_{c}\right)\left(C \Gamma^{c}\right)_{\alpha \beta}+\frac{5}{3} \epsilon_{a b c} C_{\alpha \beta} Z^{c}+\frac{2}{3} Z_{(a \mid}\left(C \Gamma_{\mid b)}\right)_{\alpha \beta} .
\end{aligned}
$$

This algebra, which we will denote as $\widetilde{\mathfrak{h m}}_{(4)}$, corresponds to a nonstandard hyper-Maxwell algebra where $P_{a b c}$ and $J_{a b c}$ transform into a spin-3 irreducible representation of the Lorentz group. We refer to this algebra as nonstandard since the translational generators $P_{a}$ are not expressed as bilinear expressions of fermionic generators $Q_{\alpha a}$. As we will see in the next section, this feature will imply that the hypersymmetric CS action based on this algebra will describe an exotic hypersymmetric action. This is analogous to what happens in the case of the nonstandard Maxwell superalgebra introduced in [89] and to the simplest hyper-Maxwell algebra obtained in the previous section.

An alternative nonstandard hyper-Maxwell algebra can also be obtained from the $\mathfrak{o} \mathfrak{a p}(1 \mid 4) \otimes \mathfrak{s p}(4) \otimes \mathfrak{s p}(4)$ superalgebra by considering a different redefinition of the $\mathfrak{o} \mathfrak{g} \mathfrak{p}(1 \mid 4)$ and $\mathfrak{s p}(4)$ generators:

$$
\begin{aligned}
& J_{a}=T_{a}+T_{a}^{+}+T_{a}^{-}, \quad P_{a}=\frac{1}{\ell}\left(T_{a}^{+}-T_{a}^{-}\right), \quad Z_{a}=\frac{1}{\ell^{2}}\left(T_{a}^{+}+T_{a}^{-}\right), \\
& J_{a b c}=\frac{1}{\ell}\left(T_{a b c}+T_{a b c}^{+}+T_{a b c}^{-}\right), \quad P_{a b c}=\frac{1}{\ell}\left(T_{a b c}^{+}-T_{a b c}^{-}\right), \quad Z_{a b c}=\frac{1}{\ell}\left(T_{a b c}^{+}+T_{a b c}^{-}\right), \\
& Q_{\alpha a}=\frac{\sqrt{2}}{\ell} \mathcal{G}_{\alpha a} \text {. }
\end{aligned}
$$

Such a redefinition differs from the previous one [Eq. (4.7)] at the level of the length parameter $\ell$. The set of generators $\left\{J_{a}, P_{a}, Z_{a}, J_{a b c}, P_{a b c}, Z_{a b c}, Q_{\alpha a}\right\}$ satisfies a different hypersymmetric version of the AdS-Lorentz algebra. In this case, the flat limit $\ell \rightarrow \infty$ reproduces a different nonstandard hyper-Maxwell algebra whose nonvanishing (anti)commutators read 


$$
\begin{array}{rlrl}
{\left[J_{a}, J_{b}\right]} & =\epsilon^{m}{ }_{a b} J_{m}, & {\left[J_{a}, P_{b}\right]=\epsilon^{m}{ }_{a b} P_{m},} \\
{\left[J_{a}, Z_{b}\right]} & =\epsilon^{m}{ }_{a b} Z_{m}, & {\left[P_{a}, P_{b}\right]=\epsilon^{m}{ }_{a b} Z_{m},} \\
{\left[J_{a}, J_{b c d}\right]} & =3 \epsilon^{m}{ }_{a(b} J_{c d) m}, & {\left[J_{a}, P_{b c d}\right]=3 \epsilon^{m}{ }_{a(b} P_{c d) m},} \\
{\left[J_{a}, Z_{b c d}\right]} & =3 \epsilon^{m}{ }_{a(b} Z_{c d) m}, & \\
{\left[J_{a}, Q_{\alpha b}\right]} & =\frac{1}{2}\left(\Gamma_{a}\right)_{\alpha}^{\beta} Q_{\beta b}+\epsilon_{a b c} Q_{\beta}^{c}, \\
{\left[J_{a b c}, Z_{m n k}\right]} & =-6\left(\eta_{(a b} \epsilon_{c)(m}^{l} \eta_{n k)}+5 \epsilon_{(m \mid(a}^{l} \delta^{d}{ }_{b} \eta_{c) \mid n} \eta_{k) d}\right) Z_{l}, \\
{\left[P_{a b c}, P_{m n k}\right]} & =-6\left(\eta_{(a b} \epsilon_{c)(m}^{l} \eta_{n k)}+5 \epsilon_{(m \mid(a}^{l} \delta_{b}^{d} \eta_{c) \mid n} \eta_{k) d}\right) Z_{l}, \\
{\left[Z_{a b c}, Z_{m n k}\right]} & =-6\left(\eta_{(a b} \epsilon_{c)(m}^{l} \eta_{n k)}+5 \epsilon_{(m \mid(a}^{l} \delta_{b}{ }_{b} \eta_{c) \mid n} \eta_{k) d}\right) Z_{l}, \\
\left\{Q_{\alpha a}, Q_{\beta b}\right\} & =-\frac{4}{3} \eta_{a b} Z_{c}\left(C \Gamma^{c}\right)_{\alpha \beta}+\frac{5}{3} \epsilon_{a b c} C_{\alpha \beta} Z^{c} \\
& +\frac{2}{3} Z_{(a \mid}\left(C \Gamma_{\mid b)}\right)_{\alpha \beta} .
\end{array}
$$

Interestingly, one can see that the subset $\left\{J_{a}, P_{a}\right.$, $\left.Z_{a}, Q_{\alpha a}\right\}$ defines a hyper-Maxwell subalgebra without spin-4 generators which coincides with the simplest hyperMaxwell algebra $\widehat{\mathfrak{h m}}$ obtained previously [Eq. (3.1)].

\section{HYPERSYMMETRIC EXTENSION OF THE MAXWELL GRAVITY THEORY WITH SPIN-4 GAUGE FIELDS}

In this section, we construct corresponding threedimensional CS theories that are invariant under the
hyper-Maxwell algebra $\mathfrak{h m}_{(4)}$ with spin-4 generators. As we shall see, we shall require not only the presence of spin-4 gauge fields but also the inclusion of a second Majorana spin- $\frac{5}{2}$ gauge field. For completeness, we also present the construction of a nonstandard hyper-Maxwell-CS gravity based on the $\widetilde{\mathfrak{h m}}_{(4)}$ hyperalgebra.

\section{A. $\mathfrak{h m}_{(4)}$ Chern-Simons hypergravity}

Let us first consider the CS hypergravity theory that is invariant under the hyper-Maxwell algebra $\mathfrak{h m}_{(4)}$ [Eqs. (4.4)-(4.6)], which is spanned by the set $\left\{P_{a}, J_{a}, Z_{a}, P_{a b c}, J_{a b c}, Z_{a b c}, Q_{\alpha}^{a}, \Sigma_{\alpha}^{a}\right\}$. The CS action can be constructed from the gauge field

$$
\begin{aligned}
A= & e^{a} P_{a}+\omega^{a} J_{a}+k^{a} Z_{a}+e^{a b c} P_{a b c}+\omega^{a b c} J_{a b c} \\
& +k^{a b c} Z_{a b c}+\bar{\psi}^{a} Q_{a}+\bar{\xi}^{a} \Sigma_{a}
\end{aligned}
$$

whose components are the dreibein, the (dualized) spin connection, the gravitational Maxwell field, three spin-4 gauge fields, and two Majorana spin- $\frac{5}{2}$ gauge fields. The corresponding curvature two-form reads

$$
F_{\mathfrak{h m}_{(4)}}=\hat{\mathcal{T}}^{a} P_{a}+\mathcal{R}^{a} J_{a}+\mathcal{F}_{(\xi)}^{a} Z_{a}+\hat{\mathcal{T}}^{a b c} P_{a b c}+\mathcal{R}^{a b c} J_{a b c}+\mathcal{F}_{(\xi)}^{a b c} Z_{a b c}+\hat{D} \bar{\psi}^{a} Q_{a}+D \bar{\xi}{ }^{a} \Sigma_{a}
$$

where

$$
\begin{aligned}
\hat{\mathcal{T}}^{a}= & T^{a}+30 \epsilon^{a}{ }_{b c} \omega^{b m n} e^{c}{ }_{m n}-\frac{3}{2} i \bar{\psi}_{b} \Gamma^{a} \psi^{b}, \\
\mathcal{R}^{a}= & R^{a}+15 \epsilon^{a}{ }_{b c} \omega^{b m n} \omega^{c}{ }_{m n}, \\
\mathcal{F}_{(\xi)}^{a}= & F^{a}+30 \epsilon^{a}{ }_{b c} \omega^{b m n} k^{c}{ }_{m n}+15 \epsilon^{a}{ }_{b c} e^{b m n} e^{c}{ }_{m n}-3 i \bar{\psi}_{b} \Gamma^{a} \xi^{b}, \\
\hat{\mathcal{T}}^{a b c}= & d e^{a b c}-10 \epsilon^{(a}{ }_{m n} \omega^{m k \mid b} e^{c) n}{ }_{k}+3 \epsilon^{(a}{ }_{m n} e^{m} \omega^{n \mid b c)}+3 \epsilon^{(a}{ }_{m n} \omega^{m} e^{n \mid b c)}+\frac{i}{2} \bar{\psi}^{(a} \Gamma^{\mid b} \psi^{c)}, \\
\mathcal{R}^{a b c}= & d \omega^{a b c}-5 \epsilon^{(a}{ }_{m n} \omega^{m k \mid b} \omega^{c) n}{ }_{k}+3 \epsilon^{(a}{ }_{m n} \omega^{m} \omega^{n \mid b c)}, \\
\mathcal{F}_{(\xi)}^{a b c}= & d k^{a b c}-10 \epsilon^{(a}{ }_{m n} \omega^{m k \mid b} k^{c) n}{ }_{k}-5 \epsilon^{(a}{ }_{m n} e^{m k \mid b} e^{c) n}{ }_{k}+3 \epsilon^{(a}{ }_{m n} \omega^{m} k^{n \mid b c)}+3 \epsilon^{(a}{ }_{m n} k^{m} \omega^{n \mid b c)} \\
& +3 \epsilon^{(a}{ }_{m n} e^{m} e^{n \mid b c)}+i \bar{\psi}^{(a} \Gamma^{b} \xi^{c)}, \\
\hat{D} \psi^{a}= & d \psi^{a}+\frac{3}{2} \omega^{b} \Gamma_{b} \psi^{a}-\omega_{b} \Gamma^{a} \psi^{b}-5 \omega^{b c a} \Gamma_{b} \psi_{c}, \\
D \xi^{a}= & d \xi^{a}+\frac{3}{2} \omega^{b} \Gamma_{b} \xi^{a}-\omega_{b} \Gamma^{a} \xi^{b}-5 \omega^{b c a} \Gamma_{b} \xi_{c}+\frac{3}{2} e^{b} \Gamma_{b} \psi^{a}-e_{b} \Gamma^{a} \psi^{b}-5 e^{b c a} \Gamma_{b} \psi_{c} .
\end{aligned}
$$

The fermionic fields and generators are assumed to be $\Gamma$ traceless, i.e, $\Gamma^{a} \psi_{a}=\Gamma^{a} \xi_{a}=0$ and $Q^{a} \Gamma_{a}=\Sigma^{a} \Gamma_{a}=0$. The $\mathfrak{h m m}_{(4)}$ hyperalgebra admits the following nonvanishing components of an invariant bilinear form: 


$$
\begin{aligned}
\left\langle J_{a} J_{b}\right\rangle & =\alpha_{0} \eta_{a b}, \quad\left\langle J_{a} P_{b}\right\rangle=\alpha_{1} \eta_{a b}, \\
\left\langle P_{a} P_{b}\right\rangle & =\alpha_{2} \eta_{a b}, \quad\left\langle J_{a} Z_{b}\right\rangle=\alpha_{2} \eta_{a b}, \\
\left\langle J_{a b c} J_{m n k}\right\rangle & =2 \alpha_{0}\left(5 \eta_{m(a} \eta_{b \mid n} \eta_{\mid c) k}-3 \eta_{(a b \mid} \eta_{\mid c)}\left(m \mid \eta_{\mid n k)}\right),\right. \\
\left\langle J_{a b c} P_{m n k}\right\rangle & =2 \alpha_{1}\left(5 \eta_{m(a} \eta_{b \mid n} \eta_{\mid c) k}-3 \eta_{(a b \mid} \eta_{\mid c)}\left(m \mid \eta_{\mid n k)}\right),\right. \\
\left\langle J_{a b c} Z_{m n k}\right\rangle & =2 \alpha_{2}\left(5 \eta_{m(a} \eta_{b \mid n} \eta_{\mid c) k}-3 \eta_{(a b \mid} \eta_{\mid c)}\left(m \mid \eta_{\mid n k)}\right),\right. \\
\left\langle P_{a b c} P_{m n k}\right\rangle & =2 \alpha_{2}\left(5 \eta_{m(a} \eta_{b \mid n} \eta_{\mid c) k}-3 \eta_{(a b \mid} \eta_{\mid c)}\left(m \mid \eta_{\mid n k)}\right),\right. \\
\left\langle Q_{\alpha a} Q_{\beta b}\right\rangle & =\frac{2 \alpha_{1}}{3}\left(2 C_{\alpha \beta} \eta_{a b}-\epsilon_{a b c}\left(C \Gamma^{c}\right)_{\alpha \beta}\right), \\
\left\langle Q_{\alpha a} \Sigma_{\beta b}\right\rangle & =\frac{2 \alpha_{2}}{3}\left(2 C_{\alpha \beta} \eta_{a b}-\epsilon_{a b c}\left(C \Gamma^{c}\right)_{\alpha \beta}\right) .
\end{aligned}
$$

Here $\alpha_{0}, \alpha_{1}$, and $\alpha_{2}$ are arbitrary constants which are related to the $\mathfrak{o} \mathfrak{s p}(1 \mid 4)$ and $\mathfrak{s p}(4)$ constants given by $(\nu, \rho)$ and $\mu$, respectively. Indeed, the invariant tensor (5.4) can be obtained from the invariant tensor of the $\mathfrak{o} \mathfrak{s} \mathfrak{p}(1 \mid 4) \otimes$ $\mathfrak{o} \mathfrak{s p}(1 \mid 4) \otimes \mathfrak{s} \mathfrak{p}(4)$ superalgebra by considering the redefinition (4.3) along with

$$
\alpha_{0}=\frac{\mu+\nu+\rho}{2}, \quad \alpha_{1}=\frac{\nu-\rho}{2 \ell}, \quad \alpha_{2}=\frac{\nu+\rho}{2 \ell^{2}},
$$

and the flat limit $\ell \rightarrow \infty$.

If one replaces the gauge connection one-form (5.1) and the invariant tensor (5.4) in the general expression for a CS action (2.7), it then reduces, up to a surface term, to

$$
\begin{aligned}
I_{\mathfrak{h m}_{(4)}}= & \frac{k}{4 \pi} \int \alpha_{1}\left(2 \mathcal{R}^{a} e_{a}+20 \mathcal{R}^{a b c} e_{a b c}+2 i \bar{\psi}_{a} \hat{D} \psi^{a}\right) \\
& +\alpha_{2}\left(2 \mathcal{R}^{a} k_{a}+e^{a} \mathcal{T}_{a}+20 \mathcal{R}^{a b c} k_{a b c}+10 e^{a b c} \mathcal{T}_{a b c}\right. \\
& \left.+2 i \bar{\psi}_{a} D \xi^{a}+2 i \bar{\xi}_{a} \hat{D} \psi^{a}\right)+\alpha_{0} L(\Omega),
\end{aligned}
$$

where $\mathcal{T}^{a}$ and $\mathcal{T}^{a b c}$ are given by

$$
\begin{aligned}
\mathcal{T}^{a}= & T^{a}+30 \epsilon^{a}{ }_{b c} \omega^{b m n} e^{c}{ }_{m n}, \\
\mathcal{T}^{a b c}= & d e^{a b c}-10 \epsilon^{(a}{ }_{m n} \omega^{m k \mid b} e^{c) n}{ }_{k}+3 \epsilon^{(a}{ }_{m n} e^{m} \omega^{n \mid b c)} \\
& +3 \epsilon^{(a}{ }_{m n} \omega^{m} e^{n \mid b c)},
\end{aligned}
$$

and

$$
\begin{aligned}
L(\Omega)= & L(\omega)+10\left(d \omega^{a b c}-\frac{10}{3} \epsilon^{a}{ }_{m n} \omega^{m k b} \omega_{k}^{c n}\right. \\
& \left.+3 \epsilon^{a}{ }_{m n} \omega^{m} \omega^{n b c}\right) \omega_{a b c},
\end{aligned}
$$

with $L(\omega)$ being the Lorentz-CS form (2.9).

The CS action (5.6) is invariant under the $\mathfrak{h m m}_{(4)}$ hyperalgebra and is split into three independent sectors proportional to the three arbitrary constants. The piece along $\alpha_{0}$ contains the parity-odd Lorentz-CS term $[87,88]$ together with its spin- 4 version. Along the $\alpha_{1}$ constant appears the Einstein-Hilbert term, contributions of the spin-4 fields, and a fermionic term. The term along $\alpha_{2}$ extends the Maxwell gravity with fermionic terms and also has spin- 4 field contributions. It is straightforward to see that the previous CS action reduces to the Maxwell-CS gravity (2.8) when the spin-4 and spin- $\frac{5}{2}$ fields are switched off. Let us note that, unlike with hyper-Poincare gravity [23], the presence of a second spinor gauge field $\xi^{a}$ is required to ensure the proper extension of the Maxwell gravity with spin- $-\frac{5}{2}$ and spin- 4 gauge fields.

The field equations for $\alpha_{2} \neq 0$ are given by the vanishing of the curvature two-form in Eq. (5.2), i.e., $F_{\mathfrak{h m}_{(4)}}=0$, whose components transform covariantly with respect to the hypersymmetry transformation laws

$$
\begin{aligned}
\delta e^{a} & =3 i \bar{\epsilon}_{b} \Gamma^{a} \psi^{b}, \quad \delta \omega^{a}=0, \quad \delta k^{a}=3 i \bar{\epsilon}_{b} \Gamma^{a} \xi^{b}+3 i \bar{\varrho}_{b} \Gamma^{a} \psi^{b}, \\
\delta e^{a b c} & =-i \bar{\epsilon}^{a} \Gamma^{b} \psi^{c}, \quad \delta \omega^{a b c}=0, \quad \delta k^{a b c}=-i \bar{\varrho}^{a} \Gamma^{b} \psi^{c}-i \bar{\epsilon}^{a} \Gamma^{b} \xi^{c}, \\
\delta \psi^{a} & =d \epsilon^{a}+\frac{3}{2} \omega^{b} \Gamma_{b} \epsilon^{a}-\omega_{b} \Gamma^{a} \epsilon^{b}-5 \omega^{b c a} \Gamma_{b} \epsilon_{c}, \\
\delta \xi^{a} & =d Q^{a}+\frac{3}{2} \omega^{b} \Gamma_{b} Q^{a}-\omega_{b} \Gamma^{a} Q^{b}-5 \omega^{b c a} \Gamma_{b} \varrho_{c}+\frac{3}{2} e^{b} \Gamma_{b} \epsilon^{a}-e_{b} \Gamma^{a} \epsilon^{b}-5 e^{b c a} \Gamma_{b} \epsilon_{c},
\end{aligned}
$$

where $\epsilon^{a}$ and $\varrho^{a}$ are the fermionic gauge parameters related to the respective $Q_{a}$ and $\Sigma_{a}$ generators.

The novel hypergravity theory can be seen as a Maxwellian generalization of the hyper-Poincaré gravity in the presence of spin- 4 gauge fields. In particular, the hyper-Poincaré-CS action coupled to spin- 4 gauge fields and its exotic counterpart appear as particular subcases along the $\alpha_{1}$ and $\alpha_{0}$ constants, respectively. Let us note that, in the absence of spin- 4 gauge fields, the term along $\alpha_{1}$ constant corresponds to the usual hypergravity introduced in $[7,23]$.
Although the $\mathfrak{h m}_{(4)}$ hyperalgebra can be seen as an enlargement and deformation of the hyper-Poincaré symmetry, the present hypergravity theory does not contain a cosmological constant term.

\section{B. Nonstandard hypersymmetric Maxwell-Chern-Simons action}

Now we shall construct the CS action that is invariant under the nonstandard hyper-Maxwell algebra in the presence of spin-4 generators given by Eqs. (4.8)-(4.10), 
which is spanned by $\left\{P_{a}, J_{a}, Z_{a}, J_{a b c}, P_{a b c}, Z_{a b c}, Q_{\alpha}^{a}\right\}$. The gauge field one-form is given by

$$
\begin{aligned}
A= & e^{a} P_{a}+\omega^{a} J_{a}+k^{a} Z_{a}+e^{a b c} P_{a b c}+\omega^{a b c} J_{a b c} \\
& +k^{a b c} Z_{a b c}+\bar{\psi}^{a} Q_{a},
\end{aligned}
$$

whose components are the dreibein, the (dualized) spin connection, the gravitational Maxwell field, three spin-4 gauge fields, and one Majorana spin-5/2 field. The curvature two-form is given in this case by

$$
\begin{aligned}
F_{\tilde{h m}_{(4)}}= & \mathcal{T}^{a} P_{a}+\mathcal{R}^{a} J_{a}+\mathcal{F}_{(\psi)}^{a} Z_{a}+\mathcal{T}^{a b c} P_{a b c} \\
& +\mathcal{R}^{a b c} J_{a b c}+\mathcal{F}_{(\psi)}^{a b c} Z_{a b c}+\hat{D} \bar{\psi}^{a} Q_{a}
\end{aligned}
$$

where $\mathcal{T}^{a}, \mathcal{T}^{a b c}$ are defined in Eq. (5.7), while $\mathcal{R}^{a}, \mathcal{R}^{a b c}$, $\hat{D} \psi^{a}$ can be found in Eq. (5.3), and

$$
\begin{aligned}
\mathcal{F}_{(\psi)}^{a}= & F^{a}+30 \epsilon^{a}{ }_{b c} \omega^{b m n} k^{c}{ }_{m n} \\
& +15 \epsilon^{a}{ }_{b c} e^{b m n} e^{c}{ }_{m n}-\frac{3}{2} i \bar{\psi}_{b} \Gamma^{a} \psi^{b}, \\
\mathcal{F}_{(\psi)}^{a b c}= & d k^{a b c}-10 \epsilon^{(a}{ }_{m n} \omega^{m k \mid b} k^{c) n}{ }_{k}-5 \epsilon^{(a}{ }_{m n} e^{m k \mid b} e^{c) n}{ }_{k} \\
& +3 \epsilon^{(a}{ }_{m n} \omega^{m} k^{n \mid b c)}+3 \epsilon^{(a}{ }_{m n} k^{m} \omega^{n \mid b c)} \\
& +3 \epsilon^{(a}{ }_{m n} e^{m} e^{n \mid b c)}+\frac{i}{2} \bar{\psi}^{(a} \Gamma^{\mid b} \psi^{c)} .
\end{aligned}
$$

The algebra $\widetilde{\mathfrak{h m}}_{(4)}$ admits the following nonvanishing components of an invariant bilinear form:

$$
\begin{aligned}
\left\langle J_{a} J_{b}\right\rangle & =\alpha_{0} \eta_{a b}, \quad\left\langle J_{a} P_{b}\right\rangle=\alpha_{1} \eta_{a b}, \\
\left\langle P_{a} P_{b}\right\rangle & =\alpha_{2} \eta_{a b}, \quad\left\langle J_{a} Z_{b}\right\rangle=\alpha_{2} \eta_{a b}, \\
\left\langle J_{a b c} J_{m n k}\right\rangle & =2 \alpha_{0}\left(5 \eta_{m(a} \eta_{b \mid n} \eta_{\mid c) k}-3 \eta_{(a b \mid} \eta_{\mid c)}\left(m \mid \eta_{\mid n k)}\right),\right. \\
\left\langle J_{a b c} P_{m n k}\right\rangle & =2 \alpha_{1}\left(5 \eta_{m(a} \eta_{b \mid n} \eta_{\mid c) k}-3 \eta_{(a b \mid} \eta_{\mid c)}\left(m \mid \eta_{\mid n k)}\right),\right. \\
\left\langle J_{a b c} Z_{m n k}\right\rangle & =2 \alpha_{2}\left(5 \eta_{m(a} \eta_{b \mid n} \eta_{\mid c) k}-3 \eta_{(a b \mid} \eta_{\mid c)}\left(m \mid \eta_{\mid n k)}\right),\right. \\
\left\langle P_{a b c} P_{m n k}\right\rangle & =2 \alpha_{2}\left(5 \eta_{m(a} \eta_{b \mid n} \eta_{\mid c) k}-3 \eta_{(a b \mid} \eta_{\mid c)}\left(m \mid \eta_{\mid n k)}\right),\right. \\
\left\langle Q_{\alpha a} Q_{\beta b}\right\rangle & =2 \alpha_{2}\left(\frac{2}{3} C_{\alpha \beta} \eta_{a b}-\frac{1}{3} \epsilon_{a b c}\left(C \Gamma^{c}\right)_{\alpha \beta}\right) .
\end{aligned}
$$

Here $\alpha_{0}, \alpha_{1}$, and $\alpha_{2}$ are arbitrary constants which are related to the $\mathfrak{o} \mathfrak{p} \mathfrak{p}(1 \mid 4)$ and $\mathfrak{s} \mathfrak{p}(4)$ constants given by $\nu$ and $(\mu, \rho)$, respectively. Indeed, the invariant tensor (5.13) can be obtained from the invariant tensor of the $\mathfrak{o} \mathfrak{s} \mathfrak{p}(1 \mid 4) \otimes$ $\mathfrak{s p}(4) \otimes \mathfrak{s p}(4)$ superalgebra by considering the redefinition (4.7) along with Eq. (5.5) and the flat limit $\ell \rightarrow \infty$.
The CS action is then given, up to boundary terms, by

$$
\begin{aligned}
I_{\tilde{h m}_{(4)}}= & \frac{k}{4 \pi} \int \alpha_{1}\left(2 \mathcal{R}^{a} e_{a}+20 \mathcal{R}^{a b c} e_{a b c}\right) \\
& +\alpha_{2}\left(2 \mathcal{R}^{a} k_{a}+e^{a} \mathcal{T}_{a}+20 \mathcal{R}^{a b c} k_{a b c}\right. \\
& \left.+10 e^{a b c} \mathcal{T}_{a b c}+2 i \bar{\psi}_{a} \hat{D} \psi^{a}\right)+\alpha_{0} L(\Omega) .
\end{aligned}
$$

The CS action based on the $\widetilde{\mathfrak{h m}}_{(4)}$ hyperalgebra describes an exotic hypersymmetric gravity theory. One can see that the spin $-\frac{5}{2}$ gauge fields do not contribute to the EinsteinHilbert sector. This is due mainly to the structure of the $\widetilde{\mathfrak{h m}}_{(4)}$ hyperalgebra (4.8)-(4.10), in which $\{Q, Q\} \nsim P$. Let us note that the same behavior appears in the simplest hyper-Maxwell gravity (3.8) and in the nonstandard supersymmetric extension of the Maxwell gravity [90]. It would be worth studying the Hietarinta version [72-74] of the nonstandard hyper-Maxwell-CS action (5.14) - in which the role of the dreibein and the gravitational Maxwell gauge field are interchanged-and its physical implications. The field equations for $\alpha_{2} \neq 0$ are given by the vanishing of the curvature two-form in Eq. (5.11), i.e., $F_{\widetilde{h m}_{(4)}}=0$. In the present case, the curvatures transform covariantly under the hypersymmetry transformation laws as follows:

$$
\begin{aligned}
\delta e^{a} & =0, \quad \delta \omega^{a}=0, \quad \delta k^{a}=3 i \bar{\epsilon}_{b} \Gamma^{a} \psi^{b}, \\
\delta e^{a b c} & =0, \quad \delta \omega^{a b c}=0, \quad \delta k^{a b c}=-i \bar{\epsilon}^{a} \Gamma^{b} \psi^{c}, \\
\delta \psi^{a} & =d \epsilon^{a}+\frac{3}{2} \omega^{b} \Gamma_{b} \epsilon^{a}-\omega_{b} \Gamma^{a} \epsilon^{b}-5 \omega^{b c a} \Gamma_{b} \epsilon_{c},
\end{aligned}
$$

where $\epsilon^{a}$ is the fermionic gauge parameter related to $Q_{a}$.

\section{CONCLUDING REMARKS}

In this work, we have presented a consistent way of coupling three-dimensional Maxwell-CS gravity theory with a spin- $\frac{5}{2}$ gauge field. To this end, we have constructed the simplest hypersymmetric extension of the Maxwell algebra, which was carried out with a consistent insertion of new fermionic generators into the three-dimensional Maxwell group which transforms into a spin- $\frac{3}{2}$ irreducible representation of the Lorentz group. The respective CS theory that is invariant under the aforementioned hyperMaxwell algebra was then introduced and can be seen as an exotic hypersymmetric gravity theory that extends the hypergravity of Aragone and Deser [7] that has allowed us to write its corresponding extension to include massless gauge fields of spin $(n+3 / 2)$. Interestingly, it has also been shown that the hypersymmetric extension of the Maxwell algebra is not unique but can be further extended to include spin-4 generators that are accompanied by additional fermionic spin- $\frac{3}{2}$ ones. Indeed, it has been shown explicitly that three different hyper-Maxwell algebras can be derived by considering the IW contraction of diverse 
combinations of the $\mathfrak{o} \mathfrak{g} \mathfrak{p}(1 \mid 4)$ and the $\mathfrak{s p}(4)$ algebras. The first of these superalgebras obtained here [Eqs. (4.4)-(4.6)] has allowed us to construct a consistent CS hypergravity theory that amounts to endowing Maxwell gravity with interacting massless spin-4 fields and two independent fermionic gauge fields of spin $\frac{5}{2}$. A second alternative hypersymmetric algebra [Eqs. (4.8)-(4.10)], named here as a nonstandard algebra, has allowed us to construct the nonstandard hypersymmetric Maxwell-CS action, which is characterized as having just one spin $-\frac{5}{2}$ fermionic gauge field. Notably, in the third case (4.12) the spin-4 gauge fields can be consistently truncated, which is consistent with the fact that the set of spin-4 generators indeed form a subalgebra, so the simplest hyper-Maxwell gravity theory that includes a single massless spin $-\frac{5}{2}$ gauge field is effectively recovered.

Note that for our simplest hyper-Maxwell-Chern-Simons gravity, as in the case of the Poincaré hypergravity [23], the inclusion of additional HS generators of half-integer spin into the Maxwell algebra does not affect the causal structure underlying Maxwell-CS gravity theory, which distinguishes it from the more familiar infinite-dimensional higher-spin algebras, which require one to extend the Lorentz group in order to include generators of higher spins $s>2$.

The results obtained here could serve as a starting point for various further studies. In particular, one could explore the possibility of including a cosmological constant in our theory. Indeed, with regard to the bosonic sector of the theory, it is encouraging to know that the Maxwell gravity theory can alternatively be recovered as a vanishing cosmological constant limit of the so-called AdS-Lorentz gravity $[70,78,94]$ and the Poincaré-Lorentz gravity theory [56]. A cosmological constant could then be incorporated in the hyper-Maxwell gravity theory by constructing a CS hypergravity theory based on a hyper-AdS-Lorentz or a hyper-Poincaré-Lorentz symmetry. Although they probably should appear as deformations of the hyper-Maxwell algebras presented here, one could expect different underlying geometries. Indeed, unlike the AdS-Lorentz gravity theory, the inclusion of a cosmological constant through the Poincaré-Lorentz symmetry implies the presence of a nonvanishing torsion [56]. It would be worth exploring the physical implications of considering a nonvanishing torsion in a hypergravity theory.

It is worth stressing that the study of suitable asymptotic symmetries for the extensions of hyper-Maxwell theories found here become crucial due to their inherent topological character, which is pointed out by the absence of local bulk degrees of freedom [86]. Thus, one can expect that suitable asymptotic conditions could be performed in each case along the lines of $[85,86]$, giving rise to adequate asymptotic symmetries being canonically realized by deformations of the hyper- $\mathfrak{b} \mathfrak{m}_{3} \mathfrak{S}_{3}$ asymptotic algebra. One might then wonder whether interesting hypersymmetry bounds could be derived from these asymptotic algebras, thereby implying prominent properties for solutions such as solitonlike ones, as well as those with a sensible thermodynamics; see, e.g., [22,23]. Indeed, since the hyper-Maxwell algebra appears as the IW contraction of diverse combinations of the $\mathfrak{o} \mathfrak{g} \mathfrak{p}(1 \mid 4)$ and the $\mathfrak{s p}(4)$ algebras, it seems natural to expect that the respective asymptotic algebras could alternatively be recovered as a precise combination of the $W_{\left(2, \frac{5}{2}, 4\right)}$ and $W_{(2,4)}$ algebras (this is a work in progress).

Another interesting aspect that deserves exploration is the derivation of the hyper-Maxwell algebras introduced here by considering the $S$-expansion method. In three spacetime dimensions, the Maxwell algebra and its supersymmetric extension can be obtained by expanding the $\mathfrak{s} \mathfrak{o}(2,1)$ and $\mathfrak{o} \mathfrak{S p}(2,1)$ algebras, respectively [70]. One could extend the $S$-expansion procedure at the hypersymmetric level by studying the $S$ expansion of the $\mathfrak{o} \mathfrak{s} \mathfrak{p}(1 \mid 4)$ superalgebra considering different semigroups. It would be interesting to recover not only known algebras (such as the hyper-Poincaré algebra and the ones presented here) but also novel algebras that are the hypersymmetric extensions of known (or unknown) bosonic algebras. A particular advantage of considering the $S$-expansion approach is that it provides us not only with the expanded (anti)commutation relations but also with the nonvanishing components of the invariant tensor of the expanded algebra, which are essentials in the construction of a CS action.

It is well known that three-dimensional CS actions possess higher-dimensional generalizations. Following [26], it would be worthwhile to extend our construction to five or more odd spacetime dimensions. As a concluding remark, let us mention that the $\mathcal{N}$ extension of our results can be done by considering an appropriate IW contraction of diverse combinations of the $\mathfrak{o} \mathfrak{g} \mathfrak{p}(M \mid 4)$ and $\mathfrak{s p}(4)$ algebras. In particular, one would expect that an $\mathcal{N}=$ $(M, N)$ extended version of the simplest hyper-Maxwell algebra presented here could be derived from the $\mathfrak{o} \mathfrak{g} \mathfrak{p}(M \mid 4) \times \mathfrak{o} \mathfrak{g} \mathfrak{p}(N \mid 4) \times \mathfrak{g} \mathfrak{p}(4)$ superalgebra. Let us note that the $\mathfrak{o} \mathfrak{g p}(M \mid 4) \times \mathfrak{o} \mathfrak{g p}(N \mid 4)$ superalgebra corresponds to the $\mathcal{N}$-extended hyper-AdS algebra studied in [22].

\section{ACKNOWLEDGMENTS}

The work of R. C., D. T., and P. C. is partially funded by the National Agency for Research and Development (ANID; formerly CONICYT) FONDECYT Grants No. 1211077, No. 1181031, No. 1181496, and No. 1211226, and Programa de Atracción e Inserción de capital humano (PAI) Grant No. 77190078. This work was supported by the Research Project Code No. DIREG_09/2020 (R. C. and P. C.) of the Universidad Católica de la Santisima Concepción, Chile. R. C. and P. C. would like to thank to the Dirección de Investigación and Vice-rectoría de Investigación of the Universidad Católica de la Santísima Concepción, Chile, for their constant support. The work of J. M. was supported by the ERC Advanced Grant "HighSpin-Grav" by FNRS-Belgium (convention FRFC PDR T.1025.14 and convention IISN 4.4503.15). 
[1] D. Z. Freedman, P. van Nieuwenhuizen, and S. Ferrara, Progress toward a theory of supergravity, Phys. Rev. D 13, 3214 (1976).

[2] S. Deser and B. Zumino, Consistent supergravity, Phys. Lett. 62B, 335 (1976).

[3] C. Aragone and S. Deser, Consistency problems of hypergravity, Phys. Lett. 86B, 161 (1979).

[4] F. A. Berends, J. W. van Holten, B. de Wit, and P. van Nieuwenhuizen, On spin 5/2 gauge fields, J. Phys. A 13, 1643 (1980).

[5] F. A. Berends, J. W. van Holten, P. van Nieuwenhuizen, and B. de Wit, On spin 5/2 gauge fields, Phys. Lett. 83B, 188 (1979); 84B, 529(E) (1979).

[6] C. Aragone and S. Deser, Higher spin Vierbein gauge fermions and hypergravities, Nucl. Phys. B170, 329 (1980).

[7] C. Aragone and S. Deser, Hypersymmetry in $D=3$ of coupled gravity massless spin 5/2 system, Classical Quantum Gravity 1, L9 (1984).

[8] B. Chen, J. Long, and Y.-N. Wang, Conical defects, black holes and higher spin (super-)symmetry, J. High Energy Phys. 06 (2013) 025.

[9] Yu. M. Zinoviev, Hypergravity in $\mathrm{AdS}_{3}$, Phys. Lett. B 739, 106 (2014).

[10] M. Henneaux, A. Perez, D. Tempo, and R. Troncoso, Hypersymmetry bounds and three-dimensional higherspin black holes, J. High Energy Phys. 08 (2015) 021.

[11] P. van Nieuwenhuizen, Three-dimensional conformal supergravity and Chern-Simons terms, Phys. Rev. D 32, 872 (1985).

[12] M. Rocek and P. van Nieuwenhuizen, $N \geq=2$ supersymmetric Chern-Simons terms as $d=3$ extended conformal supergravity, Classical Quantum Gravity 3, 43 (1986).

[13] J. H. Horne and E. Witten, Conformal Gravity in Three Dimensions as a Gauge Theory, Phys. Rev. Lett. 62, 501 (1989).

[14] U. Lindstrom and M. Rocek, Superconformal Gravity in Three Dimensions as a Gauge Theory, Phys. Rev. Lett. 62, 2905 (1989).

[15] H. Nishino and S. J. Gates, Jr., Chern-Simons theories with supersymmetries in three-dimensions, Int. J. Mod. Phys. A 08, 3371 (1993).

[16] H. Afshar, B. Cvetkovic, S. Ertl, D. Grumiller, and N. Johansson, Conformal Chern-Simons holography, Phys. Rev. D 85, 064033 (2012).

[17] M. Bertin, S. Ertl, H. Ghorbani, D. Grumiller, N. Johansson, and D. Vassilevich, Lobachevsky holography in conformal Chern-Simons gravity, J. High Energy Phys. 06 (2013) 015.

[18] H. R. Afshar, Flat/AdS boundary conditions in three dimensional conformal gravity, J. High Energy Phys. 10 (2013) 027.

[19] O. Fuentealba, H. A. González, A. Pérez, D. Tempo, and R. Troncoso, Superconformal Bondi-Metzner-Sachs Algebra in Three Dimensions, Phys. Rev. Lett. 126, 091602 (2021).

[20] S. Bellucci, S. Krivonos, and A. S. Sorin, Linearizing $W_{2,4}$ and $W B_{2}$ algebras, Phys. Lett. B 347, 260 (1995).
[21] J. M. Figueroa-O'Farrill, S. Schrans, and K. Thielemans, On the Casimir algebra of $B_{2}$, Phys. Lett. B 263, 378 (1991).

[22] M. Henneaux, A. Pérez, D. Tempo, and R. Troncoso, Extended anti-de Sitter hypergravity in $2+1$ dimensions and hypersymmetry bounds, in Proceedings of the International Workshop on Higher Spin Gauge Theories, Singapore, 2015, Vol. 101 (2015), https://www.world scientific.com/doi/abs/10.1142/9789813144101_0009.

[23] O. Fuentealba, J. Matulich, and R. Troncoso, Extension of the Poincaré group with half-integer spin generators: Hypergravity and beyond, J. High Energy Phys. 09 (2015) 003.

[24] O. Fuentealba, J. Matulich, and R. Troncoso, Asymptotically flat structure of hypergravity in three spacetime dimensions, J. High Energy Phys. 10 (2015) 009.

[25] M. Banados, R. Troncoso, and J. Zanelli, Higher dimensional Chern-Simons supergravity, Phys. Rev. D 54, 2605 (1996).

[26] O. Fuentealba, J. Matulich, and R. Troncoso, Hypergravity in five dimensions, Phys. Rev. D 101, 124002 (2020).

[27] J. Hietarinta, Supersymmetry generators of arbitrary spin, Phys. Rev. D 13, 838 (1976).

[28] H. Bacry, P. Combe, and J. Richard, Group-theoretical analysis of elementary particles in an external electromagnetic field. I. The relativistic particle in a constant and uniform field, Nuovo Cimento A 67, 267 (1970).

[29] H. Bacry, P. Combe, and J. L. Richard, Group-theoretical analysis of elementary particles in an external electromagnetic field. II. The nonrelativistic particle in a constant and uniform field, Nuovo Cimento A 70, 289 (1970).

[30] R. Schrader, The Maxwell group and the quantum theory of particles in classical homogeneous electromagnetic fields, Fortschr. Phys. 20, 701 (1972).

[31] P. Salgado and S. Salgado, $\mathfrak{g} \mathfrak{v}(D-1,1) \otimes \mathfrak{g} \mathfrak{o}(D-1,2)$ algebras and gravity, Phys. Lett. B 728, 5 (2014).

[32] F. Izaurieta, E. Rodriguez, and P. Salgado, Expanding Lie (super)algebras through Abelian semigroups, J. Math. Phys. (N.Y.) 47, 123512 (2006).

[33] J. D. Edelstein, M. Hassaine, R. Troncoso, and J. Zanelli, Lie-algebra expansions, Chern-Simons theories and the Einstein-Hilbert Lagrangian, Phys. Lett. B 640, 278 (2006).

[34] F. Izaurieta, E. Rodriguez, P. Minning, P. Salgado, and A. Perez, Standard general relativity from Chern-Simons gravity, Phys. Lett. B 678, 213 (2009).

[35] P. Concha, D. Peñafiel, E. Rodríguez, and P. Salgado, Even-dimensional general relativity from Born-Infeld gravity, Phys. Lett. B 725, 419 (2013).

[36] P. K. Concha, D. M. Penafiel, E. K. Rodriguez, and P. Salgado, Chern-Simons and Born-Infeld gravity theories and Maxwell algebras type, Eur. Phys. J. C 74, 2741 (2014).

[37] S. Bonanos and J. Gomis, Infinite sequence of Poincaré group extensions: Structure and dynamics, J. Phys. A 43, 015201 (2010).

[38] S. Bonanos and J. Gomis, A note on the ChevalleyEilenberg Cohomology for the Galilei and Poincaré Algebras, J. Phys. A 42, 145206 (2009). 
[39] J. Gomis, K. Kamimura, and J. Lukierski, Deformations of Maxwell algebra and their dynamical realizations, J. High Energy Phys. 08 (2009) 039.

[40] J. A. de Azcarraga, K. Kamimura, and J. Lukierski, Generalized cosmological term from Maxwell symmetries, Phys. Rev. D 83, 124036 (2011).

[41] R. Durka, J. Kowalski-Glikman, and M. Szczachor, Gauged AdS-Maxwell algebra and gravity, Mod. Phys. Lett. A 26, 2689 (2011).

[42] J. A. de Azcarraga, K. Kamimura, and J. Lukierski, Maxwell symmetries and some applications, Int. J. Mod. Phys. Conf. Ser. 23, 350 (2013).

[43] P. Salgado-Rebolledo, The Maxwell group in $2+1$ dimensions and its infinite-dimensional enhancements, J. High Energy Phys. 10 (2019) 039.

[44] S. Kibaroglu and O. Cebecioglu, Gauge theory of the Maxwell and semi-simple extended (anti) de Sitter algebra, arXiv:2007.14795.

[45] O. Cebecioglu and S. Kibaroglu, Maxwell-modified metric affine gravity, arXiv:2104.12670.

[46] D. Cangemi, One formulation for both lineal gravities through a dimensional reduction, Phys. Lett. B 297, 261 (1992).

[47] C. Duval, Z. Horvath, and P. A. Horvathy, Chern-Simons gravity, based on a non-semisimple group, arXiv: 0807.0977.

[48] P. Salgado, R. J. Szabo, and O. Valdivia, Topological gravity and transgression holography, Phys. Rev. D 89, 084077 (2014).

[49] T. Fukuyama and K. Kamimura, Gauge theory of twodimensional gravity, Phys. Lett. 160B, 259 (1985).

[50] K. Isler and C. A. Trugenberger, Gauge Theory of Twodimensional Quantum Gravity, Phys. Rev. Lett. 63, 834 (1989).

[51] A. H. Chamseddine and D. Wyler, Gauge theory of topological gravity in $1+1$ dimensions, Phys. Lett. B 228, 75 (1989).

[52] D. Cangemi and R. Jackiw, Gauge Invariant Formulations of Lineal Gravity, Phys. Rev. Lett. 69, 233 (1992).

[53] D. Cangemi and R. Jackiw, Poincaré gauge theory for gravitational forces in $(1+1)$ dimensions, Ann. Phys. (N.Y.) 225, 229 (1993).

[54] S. Hoseinzadeh and A. Rezaei-Aghdam, $(2+1)-$ dimensional gravity from Maxwell and semisimple extension of the Poincaré gauge symmetric models, Phys. Rev. D 90, 084008 (2014).

[55] P. Concha, N. Merino, O. Miskovic, E. Rodríguez, P. Salgado-Rebolledo, and O. Valdivia, Asymptotic symmetries of three-dimensional Chern-Simons gravity for the Maxwell algebra, J. High Energy Phys. 10 (2018) 079.

[56] H. Adami, P. Concha, E. Rodriguez, and H. R. Safari, Asymptotic symmetries of Maxwell Chern-Simons gravity with torsion, Eur. Phys. J. C 80, 967 (2020).

[57] A. Ashtekar, J. Bicak, and B. G. Schmidt, Asymptotic structure of symmetry reduced general relativity, Phys. Rev. D 55, 669 (1997).

[58] G. Barnich and G. Compere, Classical central extension for asymptotic symmetries at null infinity in three spacetime dimensions, Classical Quantum Gravity 24, F15 (2007).
[59] G. Barnich and C. Troessaert, Aspects of the BMS/CFT correspondence, J. High Energy Phys. 05 (2010) 062.

[60] G. Barnich, L. Donnay, J. Matulich, and R. Troncoso, Asymptotic symmetries and dynamics of three-dimensional flat supergravity, J. High Energy Phys. 08 (2014) 071.

[61] O. Fuentealba, J. Matulich, A. Pérez, M. Pino, P. Rodríguez, D. Tempo, and R. Troncoso, Integrable systems with $\mathrm{BMS}_{3}$ Poisson structure and the dynamics of locally flat spacetimes, J. High Energy Phys. 01 (2018) 148.

[62] R. Caroca, P. Concha, E. Rodríguez, and P. SalgadoRebolledo, Generalizing the $\mathfrak{b m g}_{3}$ and 2D-conformal algebras by expanding the Virasoro algebra, Eur. Phys. J. C 78, 262 (2018).

[63] A. F. Parsa, H. R. Safari, and M. M. Sheikh-Jabbari, On rigidity of $3 \mathrm{~d}$ asymptotic symmetry algebras, J. High Energy Phys. 03 (2019) 143.

[64] P. Concha and H. Safari, On stabilization of Maxwell-BMS algebra, J. High Energy Phys. 04 (2020) 073.

[65] R. Caroca, P. Concha, O. Fierro, E. Rodríguez, and P. Salgado-Rebolledo, Generalized Chern-Simons higherspin gravity theories in three dimensions, Nucl. Phys. B934, 240 (2018).

[66] L. Avilés, E. Frodden, J. Gomis, D. Hidalgo, and J. Zanelli, Non-relativistic Maxwell Chern-Simons gravity, J. High Energy Phys. 05 (2018) 047.

[67] P. Concha, L. Ravera, and E. Rodríguez, Three-dimensional Maxwellian extended Bargmann supergravity, J. High Energy Phys. 04 (2020) 051.

[68] P. Concha, M. Ipinza, and E. Rodríguez, Generalized Maxwellian exotic Bargmann gravity theory in three spacetime dimensions, Phys. Lett. B 807, 135593 (2020).

[69] P. Concha, L. Ravera, E. Rodríguez, and G. Rubio, Threedimensional Maxwellian extended Newtonian gravity and flat limit, J. High Energy Phys. 10 (2020) 181.

[70] P. Concha, D. M. Peñafiel, and E. Rodríguez, On the Maxwell supergravity and flat limit in $2+1$ dimensions, Phys. Lett. B 785, 247 (2018).

[71] P. Concha, $N$-extended Maxwell supergravities as ChernSimons theories in three spacetime dimensions, Phys. Lett. B 792, 290 (2019).

[72] S. Bansal and D. Sorokin, Can Chern-Simons or RaritaSchwinger be a Volkov-Akulov Goldstone?, J. High Energy Phys. 07 (2018) 106.

[73] D. Chernyavsky and D. Sorokin, Three-dimensional (higher-spin) gravities with extended Schrödinger and l-conformal Galilean symmetries, J. High Energy Phys. 07 (2019) 156.

[74] P. Concha, O. Fuentealba, J. Matulich, E. Rodríguez, and R. Troncoso, Asymptotically flat black holes and colored BMS in three dimensions (to be published).

[75] D. Chernyavsky, N. S. Deger, and D. Sorokin, Spontaneously broken $3 d$ Hietarinta/Maxwell Chern-Simons theory and minimal massive gravity, Eur. Phys. J. C 80, 556 (2020).

[76] S. Deser, R. Jackiw, and S. Templeton, Topologically massive gauge theories, Ann. Phys. (N.Y.) 140, 372 (1982); 185, 406(E) (1988). 
[77] E. Bergshoeff, O. Hohm, W. Merbis, A. J. Routh, and P. K. Townsend, Minimal massive 3D gravity, Classical Quantum Gravity 31, 145008 (2014).

[78] P. Concha, N. Merino, E. Rodríguez, P. Salgado-Rebolledo, and O. Valdivia, Semi-simple enlargement of the $\mathfrak{b} \mathfrak{m g}_{3}$ algebra from a $\mathfrak{s} \mathfrak{o}(2,2) \oplus \mathfrak{s} \mathfrak{o}(2,1)$ Chern-Simons theory, J. High Energy Phys. 02 (2019) 002.

[79] A. Campoleoni, S. Fredenhagen, S. Pfenninger, and S. Theisen, Asymptotic symmetries of three-dimensional gravity coupled to higher-spin fields, J. High Energy Phys. 11 (2010) 007.

[80] H. A. Gonzalez, J. Matulich, M. Pino, and R. Troncoso, Asymptotically flat spacetimes in three-dimensional higher spin gravity, J. High Energy Phys. 09 (2013) 016.

[81] J. Matulich, A. Perez, D. Tempo, and R. Troncoso, Higher spin extension of cosmological spacetimes in 3D: Asymptotically flat behaviour with chemical potentials and thermodynamics, J. High Energy Phys. 05 (2015) 025.

[82] M. Gary, D. Grumiller, M. Riegler, and J. Rosseel, Flat space (higher spin) gravity with chemical potentials, J. High Energy Phys. 01 (2015) 152.

[83] M. Henneaux and S.-J. Rey, Nonlinear $W_{\infty}$ as asymptotic symmetry of three-dimensional higher spin anti-de Sitter gravity, J. High Energy Phys. 12 (2010) 007.

[84] A. Campoleoni, S. Fredenhagen, and S. Pfenninger, Asymptotic $\mathcal{W}$-symmetries in three-dimensional higherspin gauge theories, J. High Energy Phys. 09 (2011) 113.

[85] M. Henneaux, A. Perez, D. Tempo, and R. Troncoso, Chemical potentials in three-dimensional higher spin anti-de Sitter gravity, J. High Energy Phys. 12 (2013) 048.

[86] C. Bunster, M. Henneaux, A. Perez, D. Tempo, and R. Troncoso, Generalized black holes in three-dimensional spacetime, J. High Energy Phys. 05 (2014) 031.

[87] A. Achucarro and P. Townsend, A Chern-Simons action for three-dimensional anti-de Sitter supergravity theories, Phys. Lett. B 180, 89 (1986).

[88] E. Witten, $(2+1)$-dimensional gravity as an exactly soluble system, Nucl. Phys. B311, 46 (1988).

[89] J. Lukierski, Generalized Wigner-Inönü contractions and Maxwell (super)algebras, Proc Steklov Inst Math / Trudy Matematicheskogo instituta imeni VA Steklova 272, 183 (2011).

[90] P. Concha, R. Durka, and E. Rodríguez, Resonant superalgebras and $\mathcal{N}=1$ supergravity theories in three spacetime dimensions, Phys. Lett. B 808, 135659 (2020).
[91] E. Inonu and E. P. Wigner, On the contraction of groups and their representations, Proc. Natl. Acad. Sci. U.S.A. 39, 510 (1953).

[92] D. V. Soroka and V. A. Soroka, Tensor extension of the Poincaré algebra, Phys. Lett. B 607, 302 (2005).

[93] D. V. Soroka and V. A. Soroka, Semi-simple extension of the (super) Poincaré algebra, Adv. High Energy Phys. 2009, 234147 (2009).

[94] J. Diaz, O. Fierro, F. Izaurieta, N. Merino, E. Rodriguez, P. Salgado, and O. Valdivia, A generalized action for $(2+1)-$ dimensional Chern-Simons gravity, J. Phys. A 45, 255207 (2012).

[95] P. Concha and E. Rodríguez, Generalized pure Lovelock gravity, Phys. Lett. B 774, 616 (2017).

[96] P. Concha and E. Rodríguez, Non-relativistic gravity theory based on an enlargement of the extended Bargmann algebra, J. High Energy Phys. 07 (2019) 085.

[97] R. D'Auria and P. Fre, Geometric supergravity in $d=11$ and its hidden supergroup, Nucl. Phys. B201, 101 (1982); B206, 496(E) (1982).

[98] M. B. Green, Supertranslations, superstrings and ChernSimons forms, Phys. Lett. B 223, 157 (1989).

[99] S. Bonanos, J. Gomis, K. Kamimura, and J. Lukierski, Maxwell Superalgebra and Superparticle in Constant Gauge Backgrounds, Phys. Rev. Lett. 104, 090401 (2010).

[100] S. Bonanos, J. Gomis, K. Kamimura, and J. Lukierski, Deformations of Maxwell superalgebras and their applications, J. Math. Phys. (N.Y.) 51, 102301 (2010).

[101] J. de Azcarraga and J. Izquierdo, Minimal $D=4$ supergravity from the superMaxwell algebra, Nucl. Phys. B885, 34 (2014).

[102] P. Concha and E. Rodríguez, Maxwell superalgebras and Abelian semigroup expansion, Nucl. Phys. B886, 1128 (2014).

[103] P. Concha and E. Rodríguez, $N=1$ supergravity and Maxwell superalgebras, J. High Energy Phys. 09 (2014) 090.

[104] D. M. Peñafiel and L. Ravera, On the hidden Maxwell superalgebra underlying $D=4$ supergravity, Fortschr. Phys. 65, 1700005 (2017).

[105] L. Ravera, Hidden role of Maxwell superalgebras in the free differential algebras of $D=4$ and $D=11$ supergravity, Eur. Phys. J. C 78, 211 (2018).

[106] P. Concha, L. Ravera, and E. Rodríguez, On the supersymmetry invariance of flat supergravity with boundary, J. High Energy Phys. 01 (2019) 192. 